\title{
Noise and Dissipation in Quantum Theory
}

\author{
Luigi Accardi ${ }^{1}$ \\ Center for Mathematical System Theory \\ Department of Mathematics \\ University of Florida
}

\footnotetext{
${ }^{1}$ On leave of absence from Centro Matematico V.Volterra, Dipartimento di Matematica, Universita' di Roma II
} 


\section{Contents}

1 Introduction 3

2 Statement of the problem and description of the results 8

3 Wave automorphism, cocycles and quantum flows 12

4 Connection with scattering theory $\quad 14$

5 Notations 18

6 Local states and local algebras $\quad 20$

7 A hierarchy of chaotic systems 22

8 The Kinematics of quantum stochastic motions 27

9 The dynamics of quantum stochastic motions 29

10 Quantum diffusions $\quad 41$

11 The Forward Langevin equation 42

12 The forward master equation and the quantum Feynman-Kac formula $\quad 44$

13 Standard forms of the quantum Langevin equation 50

14 Standard forms of the quantum master equation 54

15 The damped harmonic oscillator $\quad 57$

16 The angular momentum Lie algebra $\quad 59$ 


\begin{abstract}
.
A model independent generalization of usual quantum mechanics, including the usual as well as the dissipative quantum systems, is proposed. The theory is developed deductively from the basic principles of the standard quantum theory, the only new qualitative assumption being that we allow the wave operator at time $t$ of a quantum system to be non differentiable (in $t$ ) in the usual sense, but only in an appropriately defined (Section (5.) ) stochastic sense. The resulting theory is shown to lead to a natural generalization of the usual quantum equations of motion, both in the form of the Schrödinger equation in interaction representation (Section (6.) ) and of the Heisenberg equation (Section (8.) ). The former equation leads in particular to a quantum fluctuation-dissipation relation of Einstein's type. The latter equation is a generalized Langevin equation, from which the known form of the generalized master equation can be deduced via the quantum Feynman-Kac technique (Sections (9.) and (10.)) ). For quantum noises with increments commuting with the past the quantum Langevin equation defines a closed system of (usually nonlinear) stochastic differential equations for the observables defining the coefficients of the noises. Such systems are parametrized by certain Lie algebras of observables of the system (Section (10.) ). With appropriate choices of these Lie algebras one can deduce generalizations and corrections of several phenomenological equations previously introduced at different times to explain different phenomena . Two examples are considered : the Lie algebra $[q, p]=i$ (Section (12.)) , which is shown to lead to the equations of the damped harmonic oscillator ; and the Lie algebra of $S O(3)$ ) (Section (13.)) which is shown to lead to the Bloch equations . In both cases the equations obtained are independent of the model of noise. Moreover, in the former case, it is proved that the only possible noises which preserve the commutation relations of $p, q$ are the quantum Brownian motions, commonly used in Laser theory and solid state physics.
\end{abstract}

\title{
1 Introduction
}

The problem of describing some mathematical model of "quantum noise" arises in the description of various quantum phenomena of dissipative nature (radiation damping, nuclear magnetic resonances, ... ). Originally (Kerner [20] and Stevens [34]) the time evolution of such systems was described by adding a dissipative term to the quantum equations of motion thus leading, 
for the harmonic oscillator, to an equation of the form:

$$
\frac{d q}{d t}=\omega p-\gamma q \quad ; \quad \frac{d p}{d t}=-\omega q-\gamma p
$$

where $\mathrm{p}(\mathrm{t})$ and $\mathrm{q}(\mathrm{t})$ are now operators which, at time zero satisfy the usual commutation relations. However, as noted by Senitzky [33], doing so the resulting time evolution was not unitary in particular for $t>0, \mathrm{q}(\mathrm{t})$ and $\mathrm{p}(\mathrm{t})$ satisy the commutation relations

$$
[q(t), p(t)]=i e^{-2 \gamma t}
$$

rather than the usual one, against one of the basic principles of quantum mechanics. Senitzky proposed to derive the quantum dissipation mechanism with the introduction of a "quantum noise " i.e., a (nonrelativistic) quantum field interacting with the oscillator. For an appropriately chosen form of the interaction, energy will flow away from the oscillator to the quantum noise field (also called "heat bath " or "resevoir" in the literature) so that, if one looks only at the observables of the oscillators, dissipation effects will be detected, while for the global system (i.e. oscillator + noise ) the unitarity of the evolution is respected. Starting from an idealized, but plausible, physical model and introducing several approximations, he was led to look for a quantum field $\pi(t)$ such that the solutions of the equations

$$
\begin{gathered}
\frac{d q}{d t}=p \\
\frac{d p}{d t}=-\omega q-\gamma p+\pi(t)
\end{gathered}
$$

on the Hilbert space $H_{o} \otimes H$-the tensor product of the oscillator space and the noise space - satisfy at each time t the commutation relations:

$$
[q(t), p(t)]=i
$$

He found an approximate solution to this problem by choosing the quantum field $\pi(t)$ to be the self-adjoint gaussian quantum field with mean zero, covariance

$$
<\pi_{s} \pi_{t}>=c_{1} \delta(t-s)+i c_{2} P \frac{1}{(t-s)}
$$

$(<\cdot>$ means average in the state of the field $)$ and commutation relations

$$
\left[\pi_{s}, \pi_{t}\right]=\kappa P \frac{1}{(t-s)}
$$


where $\mathrm{P}$ denotes the principal part, $\kappa$ is a complex constant and $c_{1}, c_{2}$ are real constants.

Using the formula

$$
\int_{0}^{\infty} e^{i \omega(t-s)} d \omega=i P \frac{1}{(t-s)}+\pi \delta(t-s)
$$

one sees that the correlations $\left\langle\pi_{s} \pi_{t}>\right.$ proposed by Senitzky are just the positive part of the classical white noise correlations. Lax [22] found an exact solution of the problem posed by Senitzky by taking the whole spectrum of the white noise. More precisely he considered the equations

$$
\begin{gathered}
\frac{d q}{d t}=\omega p-\gamma q+\phi(t) \\
\frac{d p}{d t}=-\omega q-\gamma p+\pi(t)
\end{gathered}
$$

where $\phi(t), \pi(t)$ is a pair of conjugate gaussian quantum fields with mean zero, covariance

$$
\begin{gathered}
<\phi(s) \pi(t)>=\lambda \delta(t-s) \\
<\phi(s) \phi(t)>=<\pi(s) \pi(t)>=0
\end{gathered}
$$

and commutation relations

$$
[\phi(s), \pi(t)]=\kappa \delta(t-s)
$$

and showed that, with this choice the canonical commutation relations

$$
[q(t), p(t)]=i
$$

are satisfied for each t.

Approximately at the same time Haken [16] showed that the fields $\phi, \pi$ defined above, can be deduced under plausible approximations from the usual equations describing the interaction of the quantum electromagnetic field witth the quantized oscillator (a clear exposition of Haken's argument was given by von Waldenfels in [36]).

Kubo [21] criticized the use of the above mentioned fields on the ground that the associated quantum state (of the noise field) cannot satisfy the KuboMartin-Schwinger condition which characterizes the equilibrium states. However the recent results of [5] clarify the role of the equilibrium conditions in 
the Lax-Haken type models.

A different model was proposed by Ford, Kac and Mazur [15] who, in analogy with the classical Ornstein-Uhlenbek process, considered a linear chain of harmonic oscillators in which the collective action of all the remaining oscillators are considered as a heat bath acting on the first ones. This leads to describe the quantum noise as a self-adjoint gaussian quantum field with mean zero and covariance

$$
<E_{t} E_{s}>=\frac{f}{\pi} \int_{-\infty}^{+\infty} \frac{\hbar \omega}{1-e^{-\hbar \omega / k T}} e^{i \omega(t-s)} d \omega
$$

and commutation relations

$$
\left[E_{s}, E_{t}\right]=2 i f \hbar \delta^{\prime}(t-s)
$$

This state satisfies the KMS condition with respect to the time shift. However, the analogy with the classical Ornstein-Uhlenbek process is broken by the fact that, in the quantum case, the reduced process of the first oscillator has no particularly simple or canonical structure. A deep study of this model was done by Lewis [23] and several other authors (Thomas, Maassen, Nakazawa, [27] ... -cf. [24] for a survey and references.

Streater [35] had the idea of inverting the problem and, rather than looking for a particular solution of the Senitzky problem which satisfies the KMS condition, he started from the equations (12) (13) and classifies all the gaussian solutions satisfying certain additional conditions that will not be discussed here. He proved that there are infinitely many such solutions whose spectral functions coincide for large (compared to the frequency $\omega$ ) frequencies with the spectral function of the Senitzky-Lax noise but in the region $0 \leq k \leq 2 \omega$ depend on an essentially arbitrary function. This arbitrariness was criticized by Hasegawa, Klauder, and Lakshmanan [17] who showed that, if one postulates as model for the noise a single self-adjoint quantum stochastic process $\left(W_{t}\right)$, rather than a canonical pair $\left\{\phi_{t}, \pi_{t}\right\}$ of quantum fields, then on the same assumptions as Streater's the solution to the Senitzky problem could be determined uniquely.

In conclusion, even from these simple models, we can see that, contrarily to the classical case (where there are two canonical forms of noises in terms of which essentially all the noises can be constructed ) in the quantum case there is a large arbitrariness in the choice of the models for the fundamental noises. The simplest among these models is certainly the one which emerges 
from the abo ve mentioned work of Senitzky and Lax aa a natural generalization of classical white noise. We shall follow an established notation in quantum optics and solid state physics [16], [22], calling a quantum white noise any pair of canonically conjugate Gaussian quantum fields $Q(t), P(t)$ on the real line (or on the positive half line ) with correlations

$$
\begin{aligned}
& <Q_{s} Q_{t}>=\sigma_{11} \delta(t-s) \\
& <P_{s} P_{t}>=\sigma_{22} \delta(t-s) \\
& <Q_{s} P_{t}>=\sigma_{12} \delta(t-s)
\end{aligned}
$$

and satisfying the commutation relations

$$
[Q(s), P(t)]=i \lambda \delta(s-t)
$$

for some complex constants $\sigma_{i j}(\mathrm{i}, \mathrm{j}=1,2)$ and a real constant $\lambda$ (the identities (17) being meant in the distribution sense ). The corresponding integrated pairs

$$
Q\left(\chi_{[0, t]}\right)=\int_{0}^{t} Q(s) d s \quad ; \quad P\left(\chi_{[0, t]}\right)=\int_{0}^{t} P(s) d s
$$

$\left(\chi_{[0, t]}=0\right.$ if $s \notin[0, t]$ and $=1$ if $\left.s \in[0, t]\right)$ are called quantum brownian motions .

From the mathematical point of view the the Senitzky-Lax white noise is a particular non relativistic free boson field and from the works of Segal it had become clear that the notion of free boson field was a distribution generalization of the Wiener process and the calculus on these fields distributios, a generalization of Ito calculus (Hudson and Streater [18] took advantage of the particularly simple form of the covarianc of the Senitzky-Lax white noise and remarked that, in this case the procedure of Wick ordering reduces exactly to the classical Ito formula for polynomials. Hudson and Parthasarathy [19] systematically developed a stochastic calculus for this model of quantum white noise. All the results mentioned above depend very strongly on the

choice of some specific models of the perturbed system, the noise, the coupling of the system with the noise... ) and it is not clear a priori to what extent they reflect a universal mechanism of quantum dissipation.

A natural way to clarify this point is to try and develop a model independent analysis of the mechanism of quantum noise and quantum dissipation in analogy with the analysis of classical stochastic motions [28]. This program 
is particularly well suited for quantum theory, in fact, while in classical mechanics the stochastic description should be in some sense superimposed to the classical, deterministic description, quantum theory is stochastic by itself and we only have to distinguish the kind of stochasticity that is peculiar to what we would call quantum noise from the generic stochasticity which is common to every quantum system (cf. Section (4. below for a mathematical formulation of this distinction). In other terms, we do not want to separate a priori a "system" in interaction with a "noise", but within a single quantum system we want to separate two time scales at which its dynamical evolution takes place: a slow scale, corresponding to the deterministic motions and a fast scale, corresponding to the stochastic motions. To achieve this separation, we shall extend to a quantum theoretical context some well known tools of classical stochastic analysis. It is somewhat surprising that, starting from such abstract qualitative premises, one is led to the unification of several phenomenological equations, introduced at different times and for different purposes by several authors and, what is maybe more interesting, to the individuation of a general mechanism which allows to produce a whole new class of such equations.

In this sense we might refer to this program as to "Quantum Stochastic Mechanics".

\section{Statement of the problem and description of the results}

In this Section we recall some known facts about the interaction representation.

The study of a single Hamiltonian $H_{o}$ is mathematically equivalent to the study of the unitary evolution generated by it:

$$
V_{t}^{o}=e^{i t H_{o}}
$$

If we think of $H_{o}$ as the free Hamiltonian of some quantum system and if we want to study perturbations of the free Hamiltonian of the form:

$$
H=H_{o}+H_{I}
$$


a standard device is to go to the interaction picture and solve the timedependent Schrödinger equation

$$
\frac{d}{d t} U_{t}=-i H_{I}(t) U_{t} \quad ; \quad U_{o}=1
$$

where $H_{I}(t)$ is the time t evolved of the interaction Hamiltonian $H_{I}$ under the free evolution:

$$
H_{I}(t)=V_{t}^{o} H_{I} V_{-t}^{o}=u_{t}^{o}\left(H_{I}\right)
$$

and where we have introduced the shorthand notation:

$$
u_{t}^{o}(\cdot)=V_{t}^{o}(\cdot) V_{-t}^{o}
$$

The solution of $(21)$ is the wave operator at time $\mathbf{t}$, i.e.

$$
U_{t}=V_{t}^{o} V_{-t}
$$

where

$$
V_{t}:=e^{i t H}
$$

$\left(U_{t}\right)$ is a one-parameter family of unitary operators satisfying the identity:

$$
U_{s+t}=u_{s}^{o}\left(U_{t}\right) \cdot U_{s}
$$

with $u_{s}^{o}$ given by (23). A one-parameter family $\left(U_{t}\right)$ of unitary operators satisfying the identity, (26) is called a left unitary $u_{t}^{o}$-cocycle. On the other hand, if $\left(U_{t}\right)$ is a left unitary cocycle then the one-parameter family $\left(V_{t}\right)$, defined by

$$
V_{t}=U_{t}^{*} \cdot V_{t}^{o}
$$

is a quantum dynamics, i.e. a one-parameter family of unitary operations satisfying

$$
V_{s+t}=V_{s} \cdot V_{t}
$$

If $U_{t}$ is strongly continuous, then the one parameter unitary group $V_{t}$ is strongly continuous hence, by Stone' s theorem, differentiable on a dense domain and therefore it has the form (25) for some self-adjoint operator $\mathrm{H}$.

Moreover, if $U_{t}$ is differentiable, then it satisfies the Schrödinger equation (21) with $H_{I}(t)$ given by $(22)$ and

$$
-i H_{I}=\left.\frac{d}{d t}\right|_{t=0} U_{t}
$$


On this assumption the interacting dynamics $V_{t}$ has the form (20). A crucial remark for what follows is that only the strong continuity of $U_{t}$ is needed for the differentiability of $V_{t}$, i.e. for the existence of the interacting Hamiltonian $\mathrm{H}$, while the identity (20) requires also the strong differentiability of $U_{t}$. Summing up: The study of all the possible perturbations $H_{I}$ of a given free Hamiltonian $H_{o}$ is mathematically equivalent to the study of all the differentiable left unitary $u_{t}^{o}$-cocycles $U_{t}$, with $u_{t}^{o}$ given by (2), (23). Any such cocycle satisfies the Schrödinger equation in interaction representation (21) with $H_{I}(t)$ given by $(22),(29)$. Conversely, once the free evolution $u_{t}^{o}$ is given, any strongly continuous cocycle $U_{t}$ defines an interacting evolution by

$$
u_{t}=U_{t}^{*} \cdot u_{t}^{o}(\cdot) \cdot U_{t}
$$

and, if $u_{t}^{o}$ is given by $(23)$, then $u_{t}=V_{t}(\cdot) V_{t}^{*}$ with $V_{t}$ given by (27). If moreover the cocycle $U_{t}$ is strongly differentiable on a dense domain contained in the domain of $H_{o}$, then $V_{t}$ will satisfy, on this domain, the Schrödinger equation:

$$
\frac{d}{d t} V_{t}=i V_{t}\left(H_{I}+H_{o}\right)
$$

with $H_{I}, H_{o}$ given by (29), (2) respectively. If $U_{t}$ is only strongly continuous, then the interacting dynamics $V_{t}$ has still a perfectly legal Hamiltonian generator $H$, but it might happen that this Hamiltonian is not related to the free Hamiltonian via the simple relation (20). In this paper we shall investigate this possibility.

Remark. An even more general class of perturbations could be considered, namely one parameter families $j_{t}$ of $*$-automorphisms such that $u_{o}^{t} \cdot j_{t}=u_{t}$ is a one-parameter group of automorphisms. This reduces to the preceeding case when each $j_{t}$ is an inner automorphism, i.e. it has the form $j_{t}=U_{t}(\cdot) U_{t}^{*}$ for some unitary operator $U_{t}$. This more general situation has been studied in [6/d] and shall not be considered in the present paper. To the evolution $V_{t}$ we can associate the forward Heisenberg evolution

$$
X_{f}(t)=V_{t} X V_{t}^{*}
$$

and the backward Heisenberg evolution

$$
X_{b}(t)=V_{t}^{*} X V_{t}
$$


of an arbitrary observable $X$. In the present context the two evolutions differ only for the sign of the Hamiltonian, but in the generalization we are going to consider the difference will be substantial. The root of this difference can be somewhat anticipated by spelling out the derivation of the equation for the two evolutions. In fact differentiating in $t$ the backward equation (32) one obtains

$$
\frac{d}{d t} X_{b}(t)=\left[-i H_{o}, X_{b}(t)\right]+\left[-i u_{-t}^{o}\left(H_{I}(t)\right), X_{b}(t)\right]
$$

which, in view of (22) yields

$$
\frac{d}{d t} X_{b}(t)=\left[-i\left(H_{o}+H_{I}\right), X_{b}(t)\right]
$$

The same argument applied to the forward evolution (31) yields

$$
\frac{d}{d t} X_{f}(t)=\left[i \tilde{H}_{o}(t), X_{f}(t)\right]+\left[i \tilde{H}_{I}(t), X_{f}(t)\right]
$$

with

$$
\tilde{H}_{o}(t)=V_{t} H_{o} V_{t}^{*} \quad ; \quad \tilde{H}_{I}(t)=V_{t} H_{I} V_{t}^{*}
$$

and since $V_{t}$ does not commute separately with $H_{o}$ and $H_{I}$ it is not true in general that

$$
\tilde{H}_{o}(t)=H_{o} \quad ; \quad \tilde{H}_{I}(t)=H_{I}
$$

but we have only

$$
\tilde{H}_{o}(t)+\tilde{H}_{I}(t)=H_{o}+H_{I}
$$

we shall come back to this remark in Section (9).

On the physical meaning of the right and lef equations

\section{Definition 1}

$$
\begin{aligned}
& U_{t}(+):=e^{i t H_{0}} e^{-i t H_{R}} \\
& U_{t}(-):=e^{-i t H_{R}} e^{i t H_{0}}
\end{aligned}
$$

Allora

$$
U_{t / \lambda^{2}}( \pm) \longrightarrow U(t, \pm)
$$

Se

$$
\begin{gathered}
H_{I}=i\left(D \otimes A^{+}(g)-D^{+} \otimes A(g)\right) \\
e^{i t H_{0}} D e^{-i t H_{0}}=e^{+i t \omega} D \quad(\omega>0)
\end{gathered}
$$

Abbiamo che 
i)

$U(t,+)=1+\int_{0}^{t}\left(D \otimes d A_{s}^{+}\left(g^{\omega}\right)-D^{+} \otimes d A_{s}\left(g^{\omega}\right)-D^{+} D \otimes 1\left(g^{\omega} \mid g^{\omega}\right)_{-} d s\right) U(s, t)$

dove

$$
\left(f^{\omega} \mid g^{\omega}\right):=\int_{-\infty}^{+\infty}\left\langle f, e^{i t H_{1}} g\right\rangle e^{i t \omega} d t
$$

ii)

$U(t,-)=1+\int_{0}^{t} U(s,-)\left(D \otimes d A_{s}^{+}\left(g_{\omega}\right)-D^{+} \otimes d A_{s}\left(g_{\omega}\right)-D^{+} D \otimes 1\left(g_{\omega} \mid g_{\omega}\right)_{-} d s\right)$

dove

$$
\left(f_{\omega} \mid g_{\omega}\right):=\int_{-\infty}^{+\infty}\left\langle f, e^{-i t H_{1}} g\right\rangle e^{-i t \omega} d t
$$

Abbiamo risultati similari in casi generali.

\section{Wave automorphism, cocycles and quan- tum flows}

Let $u_{t}^{0}, u_{t}$ be two dynamical systems. For each $t \in \mathbb{R}$, the automorphism

$$
j_{t}^{-}:=u_{t} u_{-t}^{0} \quad ; \quad t \geq 0
$$

is called the backward wave automorphism at time $t$ and the automorphism

$$
j_{t}^{+}:=u_{-t} u_{t}^{0}=j_{-t}^{-} \quad ; \quad t \geq 0
$$

is called the forward wave automorphism.

The definition (.3) has been adapted, for example, in [ReSi III]; the Definition (.1) was used in [Acnois], [AcMo1].

For two inner Heisenberg dynamics,

$$
\begin{gathered}
u_{t}^{0}(x)=V_{-t}^{o} x V_{t}^{o} ; \quad u_{t}(x)=V_{-t} x V_{t} \\
V_{t}^{o}=e^{-i t H_{o}} ; \quad V_{t}=e^{i t H}
\end{gathered}
$$


the backward wave automorphism at time $t$ is

$$
j_{t}^{-}(x)=u_{t} u_{-t}^{0}(x)=V_{-t} V_{t}^{o} x V_{-t}^{o} V_{t}=e^{i t H} e^{-i t H_{o}} x e^{i t H_{o}} e^{-i t H} ; \quad t>0
$$

Similary for the forward wave automorphism

$$
j_{t}^{+}(x)=u_{-t} u_{t}^{0}(x)=V_{t} V_{-t}^{o} x V_{t}^{o} V_{-t}=e^{-i t H} e^{-t H_{o}} x e^{-i t H_{o}} e^{i t H}
$$

Suppose that the limits

$$
\begin{aligned}
& \lim _{t \rightarrow-\infty} u_{t} u_{-t}^{0}=\lim _{t \rightarrow-\infty} j_{t}=: \omega_{+} \\
& \lim _{t \rightarrow+\infty} u_{t} u_{-t}^{0}=\lim _{t \rightarrow+\infty} j_{t}=: \omega_{-}
\end{aligned}
$$

exist in some topology and are automorphisms of $A$. Then the automorphism of $A$, defined by

$$
\sigma:=\omega_{-}^{-1} \circ \omega_{+}
$$

is called the scattering automorphism and $\omega_{+}$(resp. $\left.\omega_{-}\right)$is called the forward (resp. backward) wave automorphism.

Notice that, if the limits (??) and (??) exists in a sufficiently strong topology (e.g. the strong operator toplogy), then

$$
\begin{aligned}
& \omega_{+}(x)=\lim _{t \rightarrow-\infty} j_{t}(x)=\Omega_{+} x \Omega_{+}^{-1} \\
& \omega_{-}(x)=\lim _{t \rightarrow+\infty} j_{t}(x)=\Omega_{-} x \Omega_{-}^{-1}
\end{aligned}
$$

therefore

$$
\sigma(x)=\omega_{-}^{-1}\left(\omega_{+}(x)=\Omega_{-}^{-1} \Omega_{+} x \Omega_{+}^{-1} \Omega_{-}=S x S^{-1}\right.
$$

By construction the backward wave automorphism $j_{t}^{-}$satisfies the identity

$$
j_{t}^{-} u_{t}^{0}=u_{t}
$$

which implies

$$
u_{t+s}=j_{t+s}^{-} u_{t+s}^{0}=u_{t} u_{s}=j_{t}^{-} u_{t}^{0} j_{s}^{-} u_{s}^{0}
$$

and, since the left hand side of $(60)$ is symmetric is $(s, t)$ this is equivalent to:

$$
j_{t+s}^{-}=j_{t}^{-} u_{s}^{0} u_{-t}^{0}=j_{s}^{-} u_{s}^{0} j_{t}^{-} u_{-s}^{0}
$$


The corresponding identities for $j_{t}^{+}$is

$$
j_{t+s}^{+}=j_{t}^{+} u_{-t}^{0} j_{s}^{+} u_{t}^{0}=j_{s}^{+} u_{-s}^{0} j_{t}^{+} u_{s}^{0}
$$

Notice that also the definitions of the wave automorphism at time $t$

$$
\begin{gathered}
\tilde{j}_{t}:=u_{t}^{0} u_{-t} \\
\tilde{j}_{t}^{\prime}:=u_{-t}^{0} u_{t}=\tilde{j}_{-t}
\end{gathered}
$$

are a priori possible.

The cocycle identities for $\tilde{j}_{t}, \tilde{j}_{t}^{\prime}$ are respectively

$$
\begin{aligned}
& \tilde{j}_{t+s}=\tilde{j}_{t} u_{-t}^{0} \tilde{j}_{s} u_{t}^{0}=\tilde{j}_{s} u_{-s}^{0} \tilde{j}_{t} u_{s}^{0} \\
& \tilde{j}_{t+s}^{\prime}=\tilde{j}_{t}^{\prime} u_{t}^{0} \tilde{j}_{s}^{\prime} u_{-t}^{0}=\tilde{j}_{s}^{\prime} u_{s}^{0} \tilde{j}_{t}^{\prime} u_{-s}^{0}
\end{aligned}
$$

\section{Connection with scattering theory}

$$
U_{t}^{(\lambda)}=e^{i t H_{o}} e^{-i t H_{\lambda}}=\left(U_{t}^{(\lambda)-}\right)^{*}
$$

the notation $U_{t}^{(\lambda)-}$, in (62) has been introduced to underline that, as $t \rightarrow+\infty$ the operator $U_{t}^{(\lambda)}$, defined by (62) converges to the wave operator $\Omega_{-}^{*}$ (cf. Section (scattering)).

$j_{t}^{\lambda}$ in the limit gives the Möller wave automorphisms

$$
\lim _{t \rightarrow \mp \infty} j_{t}^{\lambda}=: j_{ \pm}^{\lambda}
$$

and the scattering automorphism is defined by

$$
\sigma^{\lambda}:=\left(j_{-}^{\lambda}\right)^{-1} \circ j_{+}^{\lambda}
$$

The wave automorphism at time $t$ (cf. Section (scatt))

$$
j_{t}^{\lambda}:=u_{t}^{\lambda} \cdot u_{-t}^{o}
$$

is an $u_{-t}^{o}-$ flow (also called an $u_{-t}^{o}-1-$ cocycle) in the sense that its time shift

$$
j_{[s, t]}^{\lambda}:=u_{s}^{o} \cdot j_{[s, t]}^{\lambda} \cdot u_{-s}^{o}
$$


satisfies the flow equation

$$
j_{[s, t]}^{\lambda} \cdot j_{[r, s]}^{\lambda}=j_{[r, t]}^{\lambda}
$$

The whole discussion above is based on the differentiability of $U_{t}$, however the differentiability of $U_{t}$ is certainly not a law of nature, but only a technical assumption useful to derive interesting equations. Therefore, if we can substitute for the differentiability of $U_{t}$ a weaker property which still allows the derivation of a class of interesting equations, this would provide $\mathbf{a}$ natural generalization of the Schrödinger equation (1.3). The goal of this paper is to realize this program and to show that the resulting equations are of considerable physical interest. The non triviality of this program and

its compatibility with the basic principles of quantum mechanics is based on the remark, already made above, that if $U_{t}$ is a left unitary $V_{t}^{o}$-cocycle such that the map $t \mapsto U_{t}$ is strongly continuous, then $V_{t}$, defined by (27) is a strongly continuous unitary group and therefore, by Stone's theorem it is also strongly differentiable on some dense domain and on it satisfies a usual Schrödinger equation

$$
\frac{d}{d t} V_{t}=i V_{t} K
$$

where $K$ is some self-adjoint operator. The basic remark is that, even if both $V_{t}$ and $V_{t}^{o}$ are differentiable, the product $V_{t}^{*}=V_{t} V_{-t}^{o}$ need not be differentiable for domain reasons. If it is, then necessarily the equations (21), (22), (29) hold and one has:

$$
K=H_{o}+H_{I}
$$

But if it isn't, then the abstract Hamiltonian K of equation (65) is related in a more complicated, non additive, way to $H_{o}$ and the following problem naturally arises:

Is it possible to describe the usual (differentiable) quantum dynamics $\left(V_{t}\right)$, associated to an arbitrary strongly continuous cocycle, not in terms of the usual Schrödinger equation (65) (on whose Hamiltonian we have no control) but of a different equation, which exploits different infinitesimal characteristics of the non-differentiable wave operator (cocycle) $U_{t}$ ?

It might happen (and in fact it happens in several interesting models of quantum optics) that these infinitesimal characteristics of the operators $U_{t}$ 
have a simple direct physical interpretation which could not be read off from the abstract Hamiltonian K.

The hint for the solution of this problem comes from the classical theory of stochastic processes. In fact, if $U_{t}$ is the wave operator at time t, defined by (24), introducing the notations:

$$
U_{t}=U_{[0, t]} \quad ; \quad u_{s}^{o}\left(U_{t}\right)=U_{[s, t+s]}
$$

the left cocycle equation (1.6) becomes equivalent to:

$$
\begin{gathered}
U_{[r, t]}=U_{[r, s]} \cdot U_{[s, t]} \quad ; \quad U_{[s, s]}=1 \\
u_{r}^{o}\left(U_{[s, t]}\right)=U_{[s+r, t+r]}
\end{gathered}
$$

In classical probability theory a 2-parameter family $\left(U_{[s, t]}\right)$ satisfying $(67)$ and (68) is called a homogeneous (more precisely: a $v_{t}^{o}$-covariant) left multiplicative functional. We extend this notion to a quantum context.

Definition 2 A two parameter family $U_{[s, t]}$ satisfying (65) is called a right multiplicative functional. If it satisfies

$$
U_{[r, t]}=U_{[s, t]} \cdot U_{[r, s]}
$$

then we speak of a left multiplicative functional. If each $U_{[s, t]}$ is unitary (resp. Hermitean) we speak of a unitary (resp. Hermitean) multiplicative functional. If the covariance condition (68) is satisfied and if $U_{[s, t]}$ is localized in $[s, t]$ in the sense to be explained in Section (3.) below, then $U_{[s, t]}$ is called a Markovian cocycle.

Generically (i.e under some regularity conditions not to be discussed here) a classical multiplicative functional is the solution of a stochastic differential equation. This suggests the program of extending to the quantum domain the analysis which, in the classical case, leads to associate a stochastic differential equation to a generic multiplicative functional, i.e. to look for a stochastic generalization of Schrödinger's equation (this, of course, should not be confused with the use of classical probabilistic techniques to solve the usual Schrödinger equation).

In Sections (2.) and (5) we introduce the quantum translation of the probabilistic notions necessary to realize this program.

In Section (4) we introduce a hierarchy of chaoticity for quantum systems which, up to some interesting peculiarity with no classical counterpart, is the 
quantum translation of the known hierarchy of ergodic type properties arising in classical statistical mechanics and in the classical theory of stationary stochastic processes.

In Sections (5) and (7) this machinery is put to work to deduce the form of the announced quantum stochastic generalization of the Schrödinger equation (21) and to show that, for systems which do not exhibit a chaotic behaviour (i.e. which are very "low" in the hierarchy of chaoticity) this equation reduced to the usual one, i.e. (21). For systems which are "high enough" in the hierarchy of chaoticity, the difference is quite substantial and in particular implies, with the introduction of the notion of forward derivative, the choice of a direction of time. The two main results of this Section are:

1.) The proof of the fact that quantum systems, sufficiently chaotic for our theory to be applicable, behave as if their motion was the resultant of two components: a deterministic one (drift) and a noisy one (called the random force or the martingale term ). The two however are not independent, being related by the quantum fluctuation-dissipation relation of point (2.) below (cf. Section (5) for a precise definition of this notion ).

2.) The proof of the quantum analogue of the Einstein relation between the random force (fluctuation) and the drift (dissipation) in the classical Langevin equation. This relation is the common forefather of all the "fluctuation-dissipation theorems" and in the quantum case it turns out to be a consequence uniquely of the unitarity condition without any equilibrium condition. A classical analogue of our derivation can be obtained for a linear random system subject to the only condition of exact conservation of energy. This suggests that also in the quantum case the root of our quantum fluctuation-dissipation result is conservation of energy and not equilibrium.

We express the result of point (1.) above by saying that "chaotic quantum systems create their own noise." To understand the meaning of this statement, one should underline that our theory is, apart from the very general and qualitative chaoticity assumption, completely model-independent: no reservoir or heat bath is put "by hand "from the exterior to create the noise.

Here the situation, from a conceptual point of view, is quite different from the classical case, where the fundamental laws of motion are supposed to be deterministic and therefore chaotic behaviour can arise only as the result of some external influence or as a very irregular deterministic motion. Since the 
quantum description of nature is statistical ab initio, the result (1.) above is not in contradiction with any of its fundamental principles: it only points out the necessity of a finer distinction among the various forms of stochasticity which can arise in quantum theory.

The next step to which Section (8) is devoted, consists in the deduction of the quantum stochastic analogue of the forward Heisenberg equation for the time $t$ evolved of an observable. This is called a forward quantum Langevin equation.

In Section (10.) we restrict our attention to a particular class of chaotic systems which roughly speaking corresponds to the case in which the increments towards the future of the random forces (martingales) are kinematically independent from (i.e. commute with) the past. For this class of systems, the quantum Langevin equation assumes a particularly simple form and it produces a closed system of equations on certain Lie algebras of observables.

Finally, in Sections (12.) and (13.) we investigate the form of these equations for two particular choises of the above mentioned Lie algebra of observables, namely :

i) The position-momentum Lie algebra.

ii) The angular momentum Lie algebra.

In case (i) we recover a generalization of the Senitzky-Lax equations for the damped harmonic oscillator [22] and we use this to prove that, on the assumption that the forward increments of the noise commute with the past, the Lax-Haken definition of the quantum white noise [16] is the only possible one compatible with the conservation at all times of the Heisenberg relations.

In case (ii) we deduce a generalization of the Bloch equations [13], [30]. The corrections to the Bloch equations , predicted by the present approach, have been discussed, from a numerical point of view, in [6/a]. A preliminary version of this paper has appeared in [4].

\section{Notations}

Let a quantum system be specified by a pair $\left\{\mathcal{H},\left(V_{t}^{o}\right)\right\}$ where $\mathcal{H}$ is a complex separable Hilbert space, the state space of the system, and $V_{t}^{o}$ is a 1-parameter unitary group on $\mathcal{H}$, called the free evolution. An additional notion is that of past filtration. By this we mean a family $\mathcal{H}_{t]}(t \in \mathbf{R})$ of 
Hilbert subspaces of $\mathcal{H}$ satisfying

$$
\begin{gathered}
s \leq t \Rightarrow \mathcal{H}_{s]} \subseteq \mathcal{H}_{t]} \subseteq \mathcal{H} \\
V_{s}^{o} \mathcal{H}_{t]}=\mathcal{H}_{s+t]}
\end{gathered}
$$

Notice that, if $e_{t]}: \mathcal{H} \rightarrow \mathcal{H}_{t]}$ denotes the orthogonal projection, then condition (71) is equivalent to

$$
V_{s}^{o} \cdot e_{t]}=e_{t+s]} \cdot V_{s}^{o}
$$

This condition is called covariance ( $V_{t}^{o}$-covariance). $\mathcal{H}_{t]}$ represents the set of all states of the system up to time $t$ and is called the past space with respect to $t$.

The space $\mathcal{H}_{t]}$ will be called the past space with respect to $t$. A stochastic process on $H$ is a family $(X(t))$ of operators on $\mathcal{H}$ (for more sophisticated definitions cf. [2] [6/b]). Such a process will be called adapted to the past filtration if for each $t$ and for each vector $\xi_{t]} \in \mathcal{H}_{t]}$ (in the domain of $X(t)$ ) one has

$$
X(t) \xi_{t]} \in \mathcal{H}_{t]}
$$

Here we shall use (73) as definition of adaptedeness, but for a more precise development, a more sophisticated notion is needed (cf. [6/c]).

This means that the observables $X(t)$ do not contain information on the history of the system in times after $t$ (causality). Of an observable, satisfying condition (73), we shall say that it is localized in $H_{t]}$.

Let $e_{t]}: \mathcal{H} \rightarrow \mathcal{H}_{t]}$ be the orthogonal projection onto the past space $(t \in \mathbf{R})$. A family of linear maps $\left(E_{t]}\right)(t \in \mathbf{R})$ from the linear operators on $\mathcal{H}$ into themselves is called a family of conditional expectations compatible with the filtration $\mathcal{H}_{t}$ if:

$$
E_{t]}(1)=1
$$

1 denoting the identity operator on $\mathcal{H}$.

$$
E_{s]} \cdot E_{t]}=E_{s]} \quad \text { if } s \leq t
$$

for each $t$ in $\mathbf{R}$ and for each vector $\xi_{t]} \in \mathcal{H}_{t]}$ one has

$$
E_{t]}(X) \cdot \xi_{t]}=e_{t]} \cdot X \cdot \xi_{t]}
$$

Notice that (2.6) with $s=t$ yields

$$
E_{t]}^{2}=E_{t]}
$$


In a certain sense $E_{t]}(X)$ represents the projection of the operator $X$ on the subspace $\mathcal{H}_{t}$.

Example (3) .

Let $\mathcal{H}, \mathcal{H}_{t]}$ be as in Example (2) above and define, for each $t$ and each operator $X$ on $\mathcal{H}$

$$
E_{t]}(X)=e_{t]} X e_{t]} \otimes 1_{[t}
$$

where $1_{[t}$ denotes the identity operator on $\Gamma\left(L^{2}((t,+\infty))\right)$ and where we have used the identification (78). Then the family $\left(E_{t]}\right)$ has the required properties and is called the family of the Fock conditional expectations.

The fundamental property of the conditional expectation $E_{t]}$ is that for any observable $X_{t}$, localized in $\mathcal{H}_{t}$, and for any observable $Y$, one has

$$
E_{t]}\left(X_{t} Y\right)=X_{t} E_{t]}(Y)
$$

heuristically this means that $E_{t]}$ acts like a functional integral on the variables localized on the future of $t$ and therefore the variables localized in the past of $t$ behave like constants with respect to $E_{t}$.

Notice that the covariance condition (3) implies that, for every $\xi_{t]} \in \mathcal{H}_{t]}$ one has

$$
V_{s}^{o} E_{t]}(X) \xi_{t]}=V_{s}^{o} e_{t]} X \xi_{t]}=e_{t+s]}\left(V_{s}^{o} X V_{s}^{o}\right) V_{s}^{o} \xi_{t]}=E_{t+s]}\left(u_{s}^{o}(X)\right) V_{s}^{o} \xi_{t]}
$$

We shall assume that, more generally

$$
u_{s}^{o} E_{t]}=E_{t+s} u_{s}^{o}
$$

which is the usual covariance condition for the conditional expectations of a stationary stochastic process.

\section{$6 \quad$ Local states and local algebras}

A classical stochastic process is a family $X(t)$ of random variables indexed by a set $\mathrm{T}$ (i.e. $t \in T$ ). In this paper we shall assume that $\mathrm{T}$ is an interval of $\mathbf{R}$ (typically $T=[0,+\infty)$ ) and the parameter $t \in T$ will be interpreted as time. Several qualitative properties of a stochastic process do not depend on the random variables $X(t)$ themselves, but only on some classes of functionals of these random variables (e.g. the polynomials in the $X(t)$, the exponentials $\exp i \lambda X(t)$, or $\exp i \int_{s}^{t} X(r) d r$, if $X(t)$ is self-adjoint. Without loss of 
generality the class of functionals of the process we are interested in can be assumed to be an algebra closed under the involution $X \mapsto X^{*}$ given by the adjoint of an operator or the formal adjoint on a given dense domain. The random variables of the process can be looked at as the generators of this algebra. When there is a set of privileged random variables, it is convenient to think of a quantum process as a family $X(t)$ of quantum observables. When such a privileged set does not exist, it is better to think of the process as a family of local algebras. In this paper we shall only be interested in time localization, so we are led to the following picture: to every interval $I \subseteq \mathbf{R}$, one associates a $*$-algebra $\mathcal{A}_{I}$ which contains all the information that one can extract from the system with measurements performed during the time interval I. This interpretation requires that

$$
I \subseteq J \Rightarrow \mathcal{A}_{I} \subseteq \mathcal{A}_{J} \quad \text { (isotony) }
$$

and we can assume that all the $\mathcal{A}_{I}$ are contained in a single algebra $\mathcal{A}$ - the algebra of all the functionals of the random variables of the process. A pair $\left\{\mathcal{A},\left(\mathcal{A}_{I}\right)\right\}$ with the above properties will be called a family of local algebras in $\mathcal{A}$. The notion of local algebra was introduced by Haag and Kastler as a natural language for relativistic quantum field theory. In [1] it was recognized that a generalization of this notion is in fact a natural language to discuss several qualitative properties of the classical, as well as quantum, stochastic process.

We will use the notations:

$$
\mathcal{A}_{t]}=\mathcal{A}_{(-\infty, t]}
$$

for the past algebra of $t$

$$
\mathcal{A}_{[t}=\mathcal{A}_{[t,+\infty)}
$$

for the future algebra of $t$ and

$$
\mathcal{A}_{t}=\mathcal{A}_{[t, t]}
$$

for the present algebra of $t(t \in \mathbf{R})$. Because of $(81)$, the family $\left(A_{t]}\right)$, called the past filtration enjoys the property

$$
s \leq t \Longrightarrow \mathcal{A}_{s]} \subseteq \mathcal{A}_{t]}
$$

and the symmetric property:

$$
s \leq t \Longrightarrow \mathcal{A}_{[s} \supseteq \mathcal{A}_{[t}
$$


holds for the future filtration $\left(\mathcal{A}_{[t}\right)$. The time evolution $u_{t}^{o}$, also called the time shift in the following, acts naturally on the local algebras and the interpretation of $\mathcal{A}_{I}$ suggests the property

$$
u_{t}^{o}\left(\mathcal{A}_{I}\right)=\mathcal{A}_{I+t} \quad(\text { time }- \text { covariance })
$$

The expectation values of observables of $\mathcal{A}$ will be taken with respect to a state $\varphi$. It is useful to realize the algebras $\mathcal{A}_{I}, \mathcal{A}$ as algebras of operators on a Hilbert space $\mathcal{H}$, called the state space of the system and the state $\varphi$ as a vector state corresponding to a unit vector $\Phi$, i.e.

$$
\varphi(X)=<\Phi, X \cdot \Phi>\quad ; \quad X \in \mathcal{A}
$$

Corresponding to the local structure on $\mathcal{A}$ there will be a local structure on $\mathcal{H}$, i.e. a family of Hilbert sub-spaces

$$
\mathcal{H}_{I} \subseteq \mathcal{H} \quad ; \quad I \subseteq \mathbf{R}
$$

satisfying

$$
I \subseteq J \Longrightarrow \mathcal{H}_{I} \subseteq \mathcal{H}_{J}
$$

The (unit) vectors in $\mathcal{H}_{I}$ correspond to the preparations of the system that can be obtained uniquely with operations performed during the time interval I. This interpretation requires the compatibility condition

$$
\mathcal{A}_{I} \cdot \mathcal{H}_{I} \subseteq \mathcal{H}_{I} \quad ; \forall I \subseteq \mathbf{R}
$$

In analogy with the past and future filtration for algebras we have the past $\left(\mathcal{H}_{t]}\right)$ and future $\left(\mathcal{H}_{[t}\right)$ filtration for Hilbert spaces. With these notations we are ready to formulate the hierarchy of chaotic properties for a general quantum system.

\section{A hierarchy of chaotic systems}

In the notations of Section (3.) we consider a quantum system defined by:

- The local algebra $\left\{\mathcal{A},\left(\mathcal{A}_{I}\right)\right\}$ of its local observables; in particular the past filtration $\left(\mathcal{A}_{t]}\right)$. - The state space $H$, a complex separable Hilbert space, and the corresponding localization $\left(H_{I}\right)$ which is compatible with $\left\{\left(\mathcal{A}_{I}\right)\right\}$ in the sense of (90). - A privileged vector state

$$
\varphi=<\Phi,(\cdot) \Phi>\quad ; \quad \Phi \in H \quad ;\|\Phi\|=1
$$


on $\mathcal{A}$ with respect to which all expectation values are going to be taken.

- A (free) dynamics, or time shift, $\left(u_{t}^{o}\right)$ for which the algebraic localization $\left(\mathcal{A}_{1}\right)$ is covariant. We shall also assume the stationarity of $\varphi$ under the free dynamics $u_{t}^{o}$, i.e.

$$
\varphi \circ u_{t}^{o}=\varphi
$$

even if for several results in the following this assumption is not necessary. The structure defined by the above listed objects will be called a quantum system or a process and now we list a hierarchy of chaoticity conditions on quantum systems (in decreasing order of chaoticity). The hierarchy is the following:

(I.) Independent increment process.

(II.) Markov processes with an expected past filtration.

(III.) Processes with an expected past-filtration.

(IV.) Processes with non deterministic Hilbert space past-filtration.

(V.) Processes with non deterministic algebraic filtration.

(VI.) Processes with deterministic Hilbert space filtration.

(VII.) Processes with deterministic algebraic filtration.

\section{Remark (1).}

This list of properties is obviously not exhaustive: even in the classical case there is a continuum of non equivalent chaoticity properties, and as we are going to explain, in the quantum case there is an even richer spectrum of possibilities. The criterion of choice for the above listed properties has been their generality, i.e. their formulation is independent of the detailed structure of the system: only the past filtration (algebraic or Hilbert space) and the state $\varphi$ is needed, and for the last four not even the state.

\section{Remark (2).}

The Properties (I.), (V.), (VII.) are a direct translation of the corresponding properties of classical stochastic processes. In the classical case, Property (IV.) coincides with property (V.); Property (VI) coincides with Property (VII); Property (III) is trivial because every filtration is expected. This implies in particular that the class defined by Property (II.) coincides with the class of all Markov processes. So we see that even at this level of generality, the peculiar structure of quantum theory introduces some chaoticity conditions which are new with respect to the classical case.

An independent increment process is one in which the algebraic structure, the Hilbert space structure and all the expectations factorize over disjoint 
intervals. More precisely:

Definition 3 A process is called Boson independent increment if: for each natural integer $n$, for each set $I_{1}, \cdots, I_{n}$ of intervals of $\mathbf{R}$ with mutually disjoint interiors one has:

$$
\begin{aligned}
\mathcal{A}_{I} & \cong \mathcal{A}_{I_{1}} \otimes \ldots \otimes \mathcal{A}_{I_{n}} \\
H_{I} & \cong H_{I_{1}} \otimes \ldots \otimes H_{I_{n}}
\end{aligned}
$$

and for any $a_{I} \cong a_{I_{1}} \otimes \ldots \otimes a_{I_{n}} \in \mathcal{A}_{I}$

$$
\varphi\left(a_{I}\right)=\varphi_{I_{1}}\left(a_{I_{1}}\right) \cdot \ldots \cdot \varphi_{I_{n}}\left(a_{I_{n}}\right)
$$

where, for any interval $I \subseteq \mathbf{R}, \varphi_{I}$ denotes the restriction of $\varphi$ on $\mathcal{A}_{I}$, i.e.

$$
\varphi_{I}=\varphi \mid \mathcal{A}_{I}
$$

In the present paper Boson independent increment processes will be simply called independent increment processes. A conditional expectation $E_{t]}$ on the past algebra $\mathcal{A}_{t]}$ corresponds to a partial expectation over the variables localized in the future of $t$. More precisely, such an expectation is characterized by the property:

$$
E_{t]}\left(a_{t]} a\right)=a_{t]} E_{t]}(a) \quad ; a_{t]} \in \mathcal{A}_{t]}, a \in \mathcal{A}
$$

and the normalization condition

$$
E_{t]}(1)=1
$$

Definition 4 An expected Markov process is a process such that for each $t$ there exists a conditional expectation $E_{t]}$ of $\mathcal{A}$ onto $\mathcal{A}_{t]}$ which is compatible with the state $\varphi$, i.e.

$$
\varphi(a)=\varphi\left(E_{t]}(a)\right.
$$

and which enjoys the Markov property, i.e.

$$
E_{t]}\left(\mathcal{A}_{[t}\right) \subseteq \mathcal{A}_{t}
$$

The free Euclidean field provides several examples of expected Markov processes which are not independent increments. 
Definition 5 A process satisfying all the conditions of Definition 4 except at most the Markov property, is called an expected process .

Definition 6 A process is said to have a nondeterministic Hilbert space filtration if for each $s<t$,

$$
H_{s]} \neq H_{t]}
$$

This means that some information on the future is not contained in the past. An extreme case of non deterministic Hilbert space filtrations are the $\mathbf{K}-$ filtrations (or filtration with the Kolmogorov property also called regular, or purely indeterministic) i.e.

$$
\bigcap_{t} H_{t]}=\{0\}
$$

(the knowledge of the state of a system at time $t$ brings no information on its remote past) or, equivalently, any memory of the past is eventually lost.

Definition 7 A system is said to have a nondeterministic algebraic filtration if, for each $s<t$, one has

$$
\mathcal{A}_{s]} \neq \mathcal{A}_{t]}
$$

An extreme case of these systems are the algebraic K-systems, characterized by the property:

$$
\bigcap_{s} \mathcal{A}_{s]}=\mathrm{C} \cdot 1
$$

Definition 8 A process is said to have a deterministic (or singular) Hilbert space (resp. algebraic) filtration if, for each $s<t$, one has

$$
H_{s]}=H_{t]}=H \quad ; \quad \operatorname{resp} . \mathcal{A}_{s]}=\mathcal{A}_{t]}=\mathcal{A}
$$

respectively

$$
\mathcal{A}_{s]}=\mathcal{A}_{t]}=\mathcal{A}
$$

(the information on the states of the system up to time $t$ is equivalent to the full information). The cyclic representation, associated to an equilibrium (or KMS) state at a given temperature has a deterministic Hilbert space filtration, but it can have a completely indeterministic algebraic filtration. 
Example (1).

Let $H$ denote the space $L^{2}(R, d t)$ and $H_{t]}$ the space $L^{2}((-\infty, t], d s)$, considered as a subspace of $H$ by putting equal to zero a function on $(t,+\infty)$. Let $\mathcal{H}=\Gamma(H)$ where, for any Hilbert space $K$, we denote $\Gamma(K)$ the Fock space over $K$. Let, for each $t, \mathcal{H}_{t]}$ denote the intersection of $\Gamma\left(H_{t]}\right)$ with the orthogonal complement in $\Gamma(H)$ of the vacuum state $\Phi$. Then the filtration $\mathcal{H}_{t]}$ is purely indeterministic. The space $H=\Gamma\left(L^{2}(R)\right)$ or $\left(\Gamma\left(L^{2}([0,+))\right)\right)$ emerged from the works of Senitzky [33], Lax [22], Haken [16], and several others, as a natural, and probably the simplest, model for the state space of quantum noise in laser theory (and more generally in the theory of dissipative quantum phenomena). It is canonically isomorphic to the $L^{2}$-space of the increments of the Wiener process (the white noise space) and its factorizing property

$$
\Gamma\left(L^{2}(R)\right) \cong \Gamma\left(L^{2}((-\infty, t])\right) \otimes \Gamma\left(L^{2}((t,+\infty))\right)
$$

corresponds to the independence of the increments of the Wiener process. We shall call $\Gamma(H)$ the state space of the Fock Brownian motion over $L^{2}(\mathbf{R})$ or simply the Fock quantum Brownian motion .

Example (2) Let $H_{o}$ be an Hilbert space and let $H, H_{t]}$ be as in Example (1). Define

$$
\mathcal{H}=H_{o} \otimes \Gamma(H) \quad ; \quad \mathcal{H}_{t]}=H_{o} \otimes \Gamma\left(H_{t]}\right)
$$

then the filtration $\mathcal{H}_{t]}$ is nondeterministic but not completely indeterministic. In fact

$$
\bigcap_{t} \mathcal{H}_{t]}=H_{o} \otimes \Phi
$$

where $\Phi$ is the vacuum state in $\Gamma(H)$ Notice that, for each t, the space $\mathcal{H}$ has the decomposition

$$
\mathcal{H} \cong \mathcal{H}_{t]} \otimes \Gamma\left(L^{2}([t,+\infty))\right)
$$

This decomposition will be often used in the following. If $H_{o}$ is interpreted as the state space of a system $S_{o}$, then $\mathcal{H}$ is interpreted as the state space of the composite system made up by $S_{o}$ and the noise, described by the Fock quantum Brownian motion. An alternative, probabilistic, interpretation is that $\Gamma(H)$ is the space of the functions of the increments of an independent increment process and $H_{o}$ is the space of the functions of the initial values of the process. 


\section{The Kinematics of quantum stochastic mo- tions}

Definition 9 A stochastic process $X(t)$ is said to admit a forward derivative with respect to the family $\left(E_{t]}\right)$ of conditional expectations, if the limit

$$
\lim _{\epsilon \rightarrow 0^{+}} E_{t]}\left(\frac{X(t+\epsilon)-X(t)}{\epsilon}\right)=D_{+} X(t)
$$

exists.

Remark The notions of limit, continuity, integral, ... used in the present paper will be referred to a topology which we leave unspecified. In many interesting examples the operators $X(t)$ are unbounded hence, in order to introduce a natural topology on them, one must take into account some domain problems (cf. [4], [6/c], [6/d] for details on this point). In practice, all the intersting topologies for these problems are given, in a quantum context, by the strong or weak operator convergence on a certain set of vectors (coherent states, polynomials of the fields applied to the vacuum, or a mixture of the two things, as in $[6 / d])$. We shall also assume that the conditional expectations $E_{t]}$ as well as operator multiplication and the involution $*$ are continuous in the topology considered. Notice that, if the past filtration is deterministic in the sense of Section (4.), then $e_{t]}$ (and therefore also $E_{t]}$ ) is the identity operator. Therefore for such systems, the forward derivative coincides with the usual derivative. More generally one has:

Lemma 1 Suppose that $X(t)$ is differentiable in the usual sense and adapted. Then, $D_{+} X(t)$ exists. If moreover the filtration $\mathcal{H}_{t]}$, is right continuous in the sense that

$$
\mathcal{H}_{t]}=\bigcap_{s \geq 0} \mathcal{H}_{t+s]}
$$

then

$$
D_{+} X(t)=\frac{d}{d t} X(t)
$$

i.e. the forward derivative coincides with the usual derivative.

Proof. The continuity of $E_{t}$ implies that, if $d X(t) / d t$ exists, then

$$
D_{+} X(t)=E_{t]}\left(\lim _{\epsilon \rightarrow 0^{+}} \frac{X(t+\epsilon)-X(t)}{\epsilon}\right)=E_{t]}\left(\frac{d X(t)}{d t}\right)
$$


Hence if (108) holds, then (1) follows from the property (72) of the conditional expectation.

According to the fundamental theorem of calculus, if a function $\mathrm{f}$ has a continuous derivative then $\mathrm{f}$ is the integral of its derivative up to an additive constant. The analogue statement for stochastic calculus (both classical and quantum) is that if a process $X(t)$ has a continuous forward (stochastic) derivative then $X(t)$ is the integral of its derivative up to an additive term which we call noise.

Theorem 1 Suppose that $X(t)$ admits a forward derivative in the sense of Definition (1) and that $D_{+} X(t)$ is continuous. Then there exists a process $M_{t}$ such that

$$
E_{s]}\left(M_{t}\right)=M_{s} \quad ; \quad s \leq t
$$

and

$$
X_{t}=X_{o}+\int_{0}^{t} D_{+} X(s) d s+M_{t}
$$

Proof. One shows that, if $D_{+} X(t)$ is continuous, then the fundamental theorem of calculus holds under the expectation sign, i.e.

$$
E_{s]}\left(X_{t}-X_{s}\right)=E_{s]}\left(\int_{s}^{t} D_{+} X(r) d r\right)
$$

$(s \leq t)$ (cf. [4], Section (3.), Corollary (2)and [6/c]). Given (112), the process $M_{t}$ defined by

$$
M_{t}=X_{t}-X_{o}-\int_{0}^{t} D_{+} X(s) d s
$$

satisfies (10) because of (112). It satisfies (111) by construction.

Definition 10 A stochastic process $\left(M_{t}\right)$ which satisfies condition (10) is called a martingale (more precisely: an $E_{t]}$-martingale).

Remark In classical probability a martingale is the standard mathematical model for a noise. For this reason we shall use interchangeably the terms noise and martingale. Example Let $\left(Y_{n}\right)$ be a sequence of mean zero

independent identically distributed classical random variables. led $E_{s}$ denote the conditional expectation relative to all the $Y_{n}$ with $n \leq s(s \in \mathbf{R})$ and let

$$
M_{t}=\sum_{n \leq t} Y_{n}
$$


Then if $s \leq t$ one has $E_{s]}\left(M_{t}\right)=M_{s}+\sum_{s<n \leq t} E_{s]}\left(Y_{n}\right)=M_{s}$ since for $n>s$ one has

$$
E_{s]}\left(Y_{n}\right)=E\left(Y_{n}\right)=0
$$

Remark Notice that if $X_{t}$ and $D_{+} X(t)$ are strongly continuous then also $M(t)$ is strongly continuous.

\section{The dynamics of quantum stochastic mo- tions}

Now we apply Theorem 1 above to the program formulated in Section (1.), i.e. to deduce an equation for the irregular unitary multiplicative functionals i.e. for those wave operators with respect to a fixed free quantum dynamics $U_{t}^{o}(\cdot)=V_{t}^{o} \cdot(\cdot) \cdot V_{-t}^{o}$, which are not differentiable in the usual sense but only in the sense of amitting a forward derivative.

Lemma 2 Let $U_{[s, t]}$ be a left (resp. right) multiplicative functional. Then the process $U_{[0, t]}=U_{t}$ admits a forward derivative if and only if for each $t$ the limit

$$
\lim _{\epsilon \rightarrow 0^{+}} E_{t]}\left(\frac{U(t, t+\epsilon)-1}{\epsilon}\right)=b(t)
$$

exists in the sense that for some operator function $b(t)$ the limit (114) exists and the equality holds. In this case the forward derivative is

$$
D_{+} U_{t}=U_{t} b(t)
$$

for a right multiplicative functional and

$$
D_{+} U_{t}=b(t) U_{t}
$$

for a left multiplicative functional.

Proof. If $U_{[s, t]}$ is a left multiplicative functional then, by the property (??) of $E_{t]}$, for each $\mathrm{t}$ and $\epsilon>0$

$$
E_{t]}\left(\frac{U_{[0, t+\epsilon]}-U_{[0, t]}}{\epsilon}\right)=E_{t]}\left(\frac{U_{[t, t+\epsilon]}-1}{\epsilon}\right) \cdot U_{[0, t]}
$$


from this (115) follows. A similar argument proves (116). Remark Notice that, if the covariance conditions (68) and (??) are fulfilled then, in the right hand side of (117) becomes

$$
u_{t}^{o} E_{0]}\left(\frac{U_{[0, \epsilon]}-1}{\epsilon}\right) \cdot U_{[0, t]}
$$

Therefore, if $\mathrm{b}$ denotes the forward derivative of $U_{t}$ at zero, i.e.

$$
b=\left.D_{+} U_{t}\right|_{t=0}
$$

the operator $b(t)$ in the equation (11) becomes

$$
b_{t}=u_{t}^{o}(b)
$$

Comparing (11) and (120) with (21) and (22), i.e. the Schrödinger equation in interaction representation, one finds that the operator $b(t)$ plays the role of the interaction Hamiltonian $H_{I}(t)$, in the interaction representation and, because of Lemma 114 above, it reduces exactly to it if $U_{t}$ is differentiable in the ordinary sense. In Theorem (2) below, we shall see that $b(t)$ is exactly equal to $H_{I}(t)$ plus an additive (damping) term, due to the noise.

By Theorem 1 and 116, if $U_{[0, t]}$ admits a continuous forward derivative then we can write

$$
U_{t}-U_{s}=\int_{s}^{t} b(r) U_{r} d r+M_{o}(s, t)
$$

where $M_{0}(t)$ is a martingale and $M_{0}(s, t)=M_{0}(t)-M_{0}(s)$. Now let us fix two positive numbers $\mathrm{t}$ and $d t$ and, for any operator valued function $F(t)$, let us introduce the notation

$$
d F(t)=d F_{t}=F(t+d t)-F(t)
$$

With this notation, (121) implies that:

$$
d U_{t}=\int_{t}^{t+d t} b_{s} U_{s} d s+d M_{o}(t)
$$

For any bounded interval $(s, t)$ we denote $P(s, t)$ the family of all finite partitions of $(s, t)$. Any such partition $P$ is determined by a natural integer $\mathrm{n}$ 
and by an ordered n-tuple $\left(t_{1}, \ldots, t_{n}\right)$ of numbers in $[s, t]$, with $t_{1}=s, t_{n}=t$ and $t_{j}<t_{j-1}$. We shall denote

$$
|P|=\max _{1 \leq j \leq n}\left(t_{j+1}-t_{j}\right)
$$

$|P|$ is called the width of the partition.

Definition 11 A two parameter operator valued function $(s, t)$ is said to be of order $o(d t)$ (small o of $\mathbf{d t}$ ) if

$$
\lim _{|P| \rightarrow 0} \sum_{j=1}^{n} G\left(t_{j}, t_{j+1}-t_{j}\right)=0
$$

Two operator valued functions $F, G$ are called equivalent if the 2-parameter operator valued function

$$
[F(t)-F(s)]-[G(t)-G(s)]
$$

is o(dt). In this case wh shall use the shorthand notation:

$$
d F \equiv d G
$$

In particular

$$
d F \equiv 0
$$

means that $F(t+d t)-F(t)$ is $o(d t)$.

Example On the Fock space $\Gamma\left(L^{2}(\mathbf{R})\right)$, considered in Example (1) of Section (4.) , consider the creation and annihilation operators

$$
A\left(\chi_{[s, t]}\right) \quad ; \quad A^{+}\left(\chi_{[s, t]}\right)
$$

where $\chi_{[s, t]}$ denotes the characteristic function of the interval $[s, t] \subseteq \mathbf{R}$ i.e.

$$
\chi_{[s, t]}=0 \quad \text { if } r \notin[s, t] \quad ; \quad=1 \text { otherwise }
$$

Then the two-parameter families

$$
A^{2}\left(\chi_{[s, t]}\right) \quad, \quad A^{+2}\left(\chi_{[s, t]}\right) \quad, \quad A^{+}\left(\chi_{[s, t]}\right) \cdot A\left(\chi_{[s, t]}\right)
$$

are of order $o(d t)$ for the topology of weak convergence on the exponential vectors

$$
\psi(f)=\Phi+\frac{f}{\sqrt{1} !}+\frac{f \otimes f}{\sqrt{2} !}+\cdots+\frac{(\otimes f)^{n}}{\sqrt{n} !}+\ldots
$$


with $\mathrm{f}$ continuous and square integrable. In fact, for any pair $\psi(f), \psi(g)$ of such vectors one has,

$$
\begin{gathered}
<\psi(f), A^{2}\left(\chi_{[t, t+d t]}\right) \psi(g)>=\left(\int_{t}^{t+d t} g d s\right)^{2}<\psi(f), \psi(g)> \\
<\psi(f), A^{+2}\left(\chi_{[t, t+d t]}\right) \psi(g)>=\left(\int_{t}^{t+d t} d s \bar{f}\right)^{2}<\psi(f), \psi(g)> \\
<\psi(f), A^{+}\left(\chi_{[t, t+d t]}\right) \cdot A\left(\chi_{[t, t+d t]}\right) \psi(g)>=\left(\int_{t}^{t+d t} \bar{f} d s\right) \cdot\left(\int_{t}^{t+d t} g d s\right)(13), \psi(g)>
\end{gathered}
$$

However $A\left(\chi_{[s, t]}\right) \cdot A^{+}\left(\chi_{[s, t]}\right)$ is not of order $o(d t)$ in the same topology since, from the Heisenberg commutation relation

$$
\left[A(f), A^{+}(g)\right]=<f, g>
$$

and from (130), one deduces that

$$
\begin{gathered}
<\psi(f), A\left(\chi_{[t, t+d t]}\right) \cdot A^{+}\left(\chi_{[t, t+d t]}\right) \psi(g)>= \\
=d t<\psi(f), \psi(g)>+\left(\int_{t}^{t+d t} \bar{f} d s\right) \cdot\left(\int_{t}^{t+d t} g d s\right)<\psi(f), \psi(g)>
\end{gathered}
$$

Remark (3). Throughout the rest of the paper we shall use the fact that if $d G(t)$ is or order $d t$ strongly on a domain $\mathrm{D}$, for example

$$
d G(t)=\int_{t}^{t+d t} F(s) d s
$$

with $F(s)$ strongly continuous on $\mathrm{D}$, and if $M^{*}(t)$ is strongly continuous on $\mathrm{D}$, then the product $d M(t) d G(t)$ is of order $o(d t)$ weakly on $\mathrm{D}$. In fact for any $\xi, \eta$ in $\mathrm{D}$;

$$
\begin{aligned}
|<\xi, d M(t) d G(g) \eta>| & \leq \text { const. }\left\|d M^{*}(t)\right\| \cdot\|d G(t) \eta\| \\
\leq & \left(\sup _{s \in[t, t+d t]}\left\|\left(M_{s}-M_{t}\right) \xi\right\|\right) d t
\end{aligned}
$$


With the same arguments one shows that, if $\mathrm{F}(\mathrm{t})$ is strongly continuous then

$$
\int_{t}^{t+d t} F(s) d s \equiv F(t) d t
$$

in the sense of (126).

Theorem 2 below and the remarks following it suggest a natural way to construct unitary cocycles with respect to a given free evolution $\left(u_{t}^{o}\right)$ namely: to solve a quantum stochastic differential equation.

Theorem 2 Let $U(s, t)$ be a strongly continuous left multiplicative functional with a continuous forward derivative. Then there exists a 2-parameter operator valued function $M(s, t)$ such that, in the notation (126)

$$
\begin{gathered}
d U_{t} \equiv(b(t) d t+M(t, t+d t)) \cdot U_{t} \\
M(r, t)=M(r, s)+M(s, t) \cdot U(r, s) \\
E_{s]}(M(s, t))=0
\end{gathered}
$$

Moreover the limits

$$
\begin{gathered}
\lim _{d t \rightarrow 0^{+}} E_{t]}\left(\frac{M^{*}(t, t+d t) \cdot M(t, t+d t)}{d t}\right)= \\
=\lim _{d t \rightarrow 0^{+}} E_{t]}\left(\frac{M(t, t+d t) \cdot M^{*}(t, t+d t)}{d t}\right)=\sigma(t)
\end{gathered}
$$

exist for each $t$ and one has:

$$
b(t)=-\frac{1}{2} \sigma(t)-i H_{I}(t)
$$

where $H_{I}(t)$ is an Hermitean operator and

$$
d M(t)=\frac{1}{2} d P(t)+i d N
$$

where

$$
d N(t)=d N(t)^{*} \quad ; \quad d P(t) \equiv d M^{*} d M-\sigma(t) d t \equiv d M d M^{*}-\sigma(t) d t
$$

Conversely, suppose that the 1-parameter family $U(s, t)$ satisfies equation (134) with b(t) and $M$ satisfying (135), (136), (137), (139), (140). Then for 
each $t, U_{t}=U_{[0, t]}$ is a unitary operator whose forward derivative exists and satisfies

$$
D_{+} U_{t}=b(t) U_{t}
$$

Moreover the martingale associated to $U_{t}$ according to Theorem (142) is

$$
M_{o}(t)=M(0, t)
$$

Proof. Using the definition 69 of left multiplicative functional and the continuity of multiplication, (121) becomes

$$
\left(U_{[t, t+d t]}-1-\int_{t}^{t+d t} b(r) U_{[t, r]} d r\right) \cdot U_{t}=M_{o}(t+d t)-M_{o}(t)
$$

Hence, defining for each $s<t$

$$
M(s, t)=\left[M_{o}(t)-M_{o}(s)\right] U_{s}^{*}
$$

one has

$M(r, s)+M(s, t) U(r, s)=\left[M_{o}(s)-M_{o}(r)\right] U_{r}^{*}+\left[M_{o}(t)-M_{o}(s)\right] U_{s}^{*}=M(r, t)$ and since $M_{o}(t)$ is a martingale:

$$
E_{s]}(M(s, t))=E_{s]}\left[M_{o}(t)-M_{o}(s)\right] U_{s}^{*}=0
$$

Using the definition (145) of $M(s, t),(123)$ becomes

$$
d U_{t}=\left[\int_{t}^{t+d t} b_{r} U_{[t, r]} d r+M(t, t+d t)\right] \cdot U_{t}
$$

now let us substitute (146) in the algebraic identity

$$
O=d\left(U^{*} U\right)(t)=d U_{t}^{*} U_{t}+U_{t}^{*} d U_{t}+d U_{t}^{*} d U_{t}
$$

(here $d U_{t}$ has the meaning specified in (122), i.e. it is a finite difference operator). Using the unitarity of $U_{t},(147)$ becomes equivalent to

$$
\begin{aligned}
0= & {\left[\int_{t}^{t+d t} U_{[t, r]}^{*} b_{r}^{*} d r+M^{*}(t, t+d t)\right]+\left[\int_{t}^{t+d t} b_{r} U_{[t, r]} d r+M(t, t+d t)\right]+} \\
& +\left[\int_{t}^{t+d t} U_{[t, r]}^{*} b_{r}^{*} d r+M^{*}(t, t+d t)\right] \cdot\left[\int_{t}^{t+d t} b_{r} U_{[t, r]} d r+M(t, t+d t)\right]
\end{aligned}
$$


Now, using Remark (3) above we have that all the products

$$
\begin{gathered}
\left(\int_{t}^{t+d t} U_{[t, r]}^{*} b_{r}^{*} d r\right) \cdot M^{*}(t, t+d t) M^{*}(t, t+d t) \cdot\left(\int_{t}^{t+d t} b_{r} U_{[t, r]} d r\right) \\
\left(\int_{t}^{t+d t} U_{[t, r]}^{*} b_{r}^{*} d r\right) \cdot\left(\int_{t}^{t+d t} b_{r} U_{[t, r]} d r\right)
\end{gathered}
$$

are of order $o(d t)$ and therefore, using (133) the notation (126), and the fact that, for each $\mathrm{t}, U_{[t, t]}=1$, we obtain

$$
\begin{aligned}
0=\int_{t}^{t+d t}\left(U_{[t, r]}^{*} b_{r}^{*}+b_{r} U_{[t, r]}\right) d r+ & M^{*}(t, t+d t) \cdot M(t, t+d t) \\
+ & M(t, t+d t)+M(t, t+d t)^{*}
\end{aligned}
$$

taking $E_{t}$-expectations of both sides of (148) and using (10) and (136) we find

$$
E_{t]}\left(M^{*}(t, t+d t) \cdot M(t, t+d t)\right) \equiv-E_{t]}\left(\int_{t}^{t+d t}\left(b_{r} U_{[t, r]}+U_{[t, r]}^{*} b_{r}^{*} d r\right)\right.
$$

From (146) and Lemma 114 we conclude that the first of the limits (137) exists and is equal to

$$
\sigma(t)=-\left(b_{t}+b_{t}^{*}\right)=-2 R e\left(b_{t}\right)
$$

Hence, denoting

$$
H_{I}(t)=\frac{1}{2 i}\left(b_{t}-b_{t}^{*}\right)=\operatorname{Im}\left(b_{t}\right)
$$

we obtain (139). Exchanging the roles of $U$ and $U^{*}$, in the identity (147), the roles of $\mathrm{M}$ and $M^{*}$ are exchanged hence the same arguments as above yield the second identity in (137). From (146) and (133) we obtain (134). Due to $U_{[t, t]}=1$, the relation (148) is equivalent to

$$
0 \equiv\left(b(t)+b^{*}(t)\right) d t+|d M(t)|^{2}+d M(t)+d M^{*}(t)
$$

Adding and subtracting to (151) the term $E_{t]}\left(|d M(t)|^{2}\right)$, and using (150) in the form:

$$
E_{t]}\left(|d M(t)|^{2}\right) \equiv \sigma(t) d t
$$

we obtain:

$$
|d M(t)|^{2}-\sigma(t) d t \equiv-\left(d M(t)+d M^{*}(t)\right)
$$


which is the first equality in (140). By considering $U_{t} U_{t}^{*}$ rather than $U_{t}^{*} U_{t}$ we obtain the symmetric equation

$$
0 \equiv d M d M^{*}-\sigma(t) d t+d M^{*}+d M
$$

and this completes the proof of (140). Conversely, let $\left(U_{t}\right)$ satisfy equation (134) with $\mathrm{M}$ and $b(t)$ satisfying $(135), \ldots,(140)$. Then taking $E_{t]}$-expectations of both sides of (134) and using (136) we see that $U_{t}$ has a forward derivative and (141) holds. Therefore by Theorem 1 we have

$$
d U(t) \equiv b(t) U(t) d t+N(t, t+d t)
$$

By (134) this implies that

$$
N(t, t+d t)=M(t, t+d t) U_{t}+o(t, d t)
$$

Therefore, if $0=t_{1}<\ldots<t_{n}$ we obtain

$$
N(t)=\sum_{j=1}^{n} N\left(t_{j}, t_{j+1}\right)=\sum_{j=1}^{n} M\left(t_{j}, t_{j+1}\right) U_{t}+\sum_{j=1}^{n} o\left(t_{j}, t_{j+1}-t_{j}\right)
$$

Using induction on (135), we see that (155) is equivalent to

$$
N(t)=M(0, t) U_{o}=M(0, t)+\sum_{j=1}^{n} o\left(t_{j}, t_{j+1}-t_{j}\right)
$$

This, in the limit $\max _{j}\left(t_{j+1}-t_{j}\right) \rightarrow 0$, yields (143). Finally,

$$
d\left(U_{t}^{*} U_{t}\right)=d U_{t}^{*} U_{t}+U_{t}^{*} d U_{t}+d U_{t}^{*} d U_{t}
$$

which, using (137), (139) and condition (153) becomes

$$
d\left(U_{t}^{*} U_{t}\right) \equiv U_{t}^{*}\left(d M^{*} d M-\sigma d t+d M^{*}+d M\right) U_{t} \equiv 0
$$

Again summing the identity (157) over a partition $\mathrm{P}$ of $(0, t)$ and going to the limit $|P| \rightarrow 0$, this yields

$$
U_{t}^{*} U_{t}=U_{o}^{*} U_{o}=1
$$

In a similar way one checks

$$
U_{t} U_{t}^{*}=1
$$


and this proves unitarity. Remark Notice that, in the proof of the unitarity condition only (134), (139), (153) have been used, in particular we did not use the existence of $E_{t]}$. Remark We want to interpret the relation (134) as a true equation. To this goal notice that the precise meaning of (134) is:

$$
U(t+d t)-U(t)=b(t) U(t) d t+M(t, t+d t) U_{t}+o(d t)
$$

with $o(t, t+d t)$ satisfying

$$
\lim _{|P| \rightarrow 0} \sum_{j=1}^{n} o\left(t_{j}, t_{j+1}-t_{j}\right)=0
$$

with $P=\left\{0=t_{1}<\ldots<t_{n}=T<\infty\right\}$ and

$$
|P|=\max _{j}\left(t_{j+1}-t_{j}\right)
$$

Now consider a fixed $t>0$ and a fixed partition $0=t_{1}<t_{2}<\cdots<t_{n}=t$ of the interval $(0, t]$, and summing the identity (37) over the increments $d t_{j}=t_{j+1}-t_{j}$, we obtain, using $U(0)=1$ :

$U_{t}-1=\sum_{j=1}^{n} b\left(t_{j}\right) U\left(t_{j}\right)\left(t_{j+1}-t_{j}\right)++\sum_{j=1}^{n} M\left(t_{j}, t_{j+1}\right) U\left(t_{j}\right)+\sum_{j=1}^{n} o\left(t_{j}, t_{j+1}-t_{j}\right)$

Using the fact that

$$
\lim _{\max _{j}\left|t_{j+1}-t_{j}\right| \rightarrow 0} \sum_{j=1}^{n} b\left(t_{j}\right) U\left(t_{j}\right)\left(t_{j+1}-t j\right)=\int_{0}^{t} b(s) U(s) d s
$$

and (160) we obtain that the limit

$$
\lim _{\max _{j}\left|t_{j+1}-t_{j}\right| \rightarrow 0} \sum_{j=1}^{n} M\left(t_{j}, t_{j+1}\right) U_{t} j=\int_{0}^{t} d M(s) U_{s}
$$

must exist. In fact from (135) and (143) we know that this limit exists trivially and is equal to $M_{o}(0, t)$. Therefore the relation (134) is equivalent to the integral equation.

$$
U_{t}=U_{s}+\int_{s}^{t} b(r) U(r) d r+\int_{s}^{t} d M(r) U(r)
$$


Theorem 3 If $U_{t}$ satisfies the cocycle equation (26), then $b(t)$ must have the form

$$
b(t)=i u_{t}^{o}\left(H_{I}\right)-\frac{1}{2} u_{t}^{o}(\sigma)
$$

for some time independent operators $H_{I}$ and $\sigma$ such that $H_{I}$ is self-adjoint and $\sigma$ is positive. Moreover

$$
M(t, t+d t)=u_{t}^{o}(M(0, d t))
$$

for each $t, d t>0$. Conversely, if $b(t)$ and $M$ satisfy the conditions (162), (163) beyond the conditions of Theorem 2, then the 1-parameter family $\left(U_{t}\right)$ is a $u_{t}^{o}$-cocycle.

Proof. Necessity: Equation (162) follows from the remark after Lemma 114. Using equations (121), (162) and the cocycle property we find

$$
\begin{aligned}
U_{t}-U_{s} & =u_{s}^{o}\left(U_{t-s}-1\right) U_{s}=u_{s}^{o}\left(\int_{0}^{t-s} b(r) U_{r} d r+M_{o}(0, t-s)\right) U_{s}= \\
& =\int_{0}^{t-s} b(s+r) u_{s}^{o}\left(U_{r}\right) U_{s} d r+u_{s}^{o}\left(M_{o}(0, t-s)\right) U_{s} \\
& =\int_{s}^{t} b(r) U(r) d r+u_{s}^{o}\left(M_{o}(0, t-s)\right) U_{s}
\end{aligned}
$$

comparing this with (121), yields $M_{o}(s, t)=u_{s}^{o}\left(M_{o}(0, t-s)\right) U_{s}$ this identity, together with (145) yields (163).

Sufficiency. The conditions of Theorem 2 assure that $U_{t}$ exists and is unitary. We have to show that, under the additional conditions (162), (163), $U_{t}$ is a $U_{t}^{o}$-cocycle. i.e. that it satisfies the identity

$$
U_{t+s}=u_{s}^{o}\left(U_{t}\right) U_{s}
$$

Now let

$$
X_{t}=U_{t+s} U_{s}^{*} \quad ; \quad Y_{t}=u_{s}^{o}\left(U_{t}\right)
$$

Then from (134), (162) and (163) we obtain

$$
\begin{gathered}
d X_{t} \equiv(b(t+s) d t+M(s+t, s+t+d t)) U_{t+s} U_{s}^{*}=u_{s}^{o}(b(t) d t+M(t, t+d t)) X_{t} \\
d Y_{t} \equiv u_{s}^{o}\left((b(t) d t+M(t, t+d t)) U_{t}\right)=u_{s}^{o}(b(t) d t+M(t, t+d t)) Y_{t}
\end{gathered}
$$

Hence $Z_{t}=X_{t}-Y_{t}$ satisfies $d Z(t) \equiv 0$. Summing over the intervals of a partition $P$, letting $|P| \rightarrow 0$ and using (125), we find $Z(t)=$ constant. Since 
$Z(0)=0$, it follows that $X(t)=Y(t)$ for each $t$, and this ends the proof. Remark. The relation (137) means that

$$
E_{t]}\left(M^{*}(t, t+d t) M(t, t+d t)\right)=E_{t]}\left(M(t, t+d t) M^{*}(t, t+d t)\right) \equiv \sigma(t) d t
$$

which suggests the order of magnitude estimate

$$
M(t, t+d t) \cong \sqrt{d} t
$$

Thus equation (134) or, equivalently (161) should be interpreted as a separation of two time scales in the evolution of $U_{t}$ : a slow, regular one, due to the term $b(t) d t$, (drift) and an irregular one of order $\sqrt{d} t$ due to the term $M(t, t+d t)$ the noise, or martingale, term. It should be underlined that up to now we have considered a single quantum system: nothing in our assumptions indicates a separation of the system into two subsystems to be identified respectively with the system and the noise (or resevoir, or heat bath,...). Thus, even if in all models constructed up to now, of the equation (134), this separation (system-reservoir) is postulated ab initio, we should interpret the general, model independent, equation (6.17), as an indication of the existence of quantum systems which "are their own noise," in the sense that, for the state space, a kinematical decomposition into a tensor product of two spaces (one related to the regular motion and the other to the noise) is impossible and yet, inside the single system, the separation between slow and fast scales of motion takes place.

Now let us assume that $U_{t}$ satisfies the cocycle equation (26) and let us form the quantum dynamics according to the prescription (27), i.e.

$$
V_{t}=U_{t}^{*} \cdot V_{t}^{o}
$$

the increment of $V_{t}$ in the interval $[t, t+d t]$ is

$$
d V_{t}=\left(d U_{t}^{*}\right) V_{t}^{o}+U_{t}^{*} d V_{t}^{o}=d U_{t}^{*} \cdot d V_{t}^{o}
$$

but $V_{t}^{o}$ is a usual quantum dynamics with generator $H_{o}$, hence

$$
d V_{t}^{o} \equiv i V_{t}^{o} H_{o} d t
$$

is of order $d t$ and $U_{t}^{*}$ is strongly continuous hence, by Remark (3) above the product $\left(d U_{t}^{*}\right)\left(d V_{t}^{o}\right)$ is of order $o(d t)$. Using (134) in (165) we obtain 
therefore

$$
\begin{aligned}
d V_{t} & \equiv U_{t}^{*}\left(b_{t}^{*} d t+d M^{*}\right) V_{t}^{o}++U_{t}^{o} V_{t}^{o}\left(i H_{o}\right) d t= \\
& =V_{t}\left(\left[\left(V_{-t}^{o} b_{t} V_{t}^{o}\right)^{*}+i H_{o}\right] d t+\left(V_{-t}^{o} d M V_{t}^{o}\right)^{*}\right)
\end{aligned}
$$

so that, using equation (139) for $b_{t}$ and denoting

$$
d M^{o}(t)=u_{-t}^{o}(d M(t))
$$

we obtain

$$
d V_{t} \equiv V_{t}\left(i\left(H_{o}+H_{I}\right) d t-\frac{1}{2} \sigma d t+d M^{o}(t)\right)
$$

On the other hand we have already seen in Section (1.) that, for any strongly continuous $U_{t}, V_{t}$ satisfies a Scrödinger equation of usual type, i.e.

$$
d V_{t} \equiv V_{t}(i K) d t
$$

for some self-adjoint K. At first sight the two relations (168) and (169) might seem in contradiction: the right hand side of (169) is of order $d t$ while, in the right hand side of (168), the dominating order is $\sqrt{d t}$ in view of the Remark above. However since we are dealing with operators both relations have a meaning only on certain domains of $H$ and therefore what the apparent contradiction means is simply that only the zero vector can lay in the intersection of the domains of validity of the two equations. Moreover, while the equation (169) is certainly mathematically correct, but does not give any insight on the relation between the free Hamiltonian and the interacting one, equation (168) is quite explicit on the relations between the generators (in the stochastic sense) of the free and the interacting evolution It is difficult to give a precise mathematical meaning to (168), even as a stochastic differential equation, since while the cocycle $U_{t}$ is adapted, the unitary evolution $V_{t}$, defined by (164), usually is not. Remark It is intuitively clear that the

operator $\sigma$ is related to a damping process and that the operator $d M^{o}(t)$ plays the role of a quantum random force. To make this intuition rigorous, we have first to deduce the generalized Langevin and the generalized master equation canonically associated to the dynamics $\left(V_{t}\right)$. 


\section{Quantum diffusions}

In the classical case not all the stochastic differential equations describe diffusions (also jump processes can arise). In this Section we want to carry this distinction into the quantum domain. To this goal, let us first consider the identities (151) of Theorem 2. Using the explicit form (145) of $M$, these can be written

$$
d M_{o}(t) U_{t}^{*}+U_{t} d M_{o}^{*}(t)-\sigma(t) d t \equiv d M(t) d M^{*}(t) \equiv d M^{*}(t) d M(t)
$$

Therefore the limits

$$
\lim _{|P| \rightarrow 0} \sum_{d t \in P} d M_{o}(t) U_{t}^{*} \quad ; \quad \lim _{|P| \rightarrow 0} \sum_{d t \in P} U_{t} d M_{o}^{*}(t)
$$

( $\mathrm{P}$ as usual denotes a partition of $(0, t)$ of width $d t$ ) exist if and only if the limits

$$
\lim _{|P| \rightarrow 0} \sum_{d t \in P} d M(t) d M^{*}(t) \quad ; \quad \lim _{|P| \rightarrow 0} \sum_{d t \in P} d M^{*}(t) d M(t)
$$

exist and in this case the limits (172) are equal. The limits (171) are denoted respectively

$$
\int_{0}^{s} d M_{o}(s) U_{s}^{*} \quad ; \quad \int_{0}^{s} U_{s} d M_{o}^{*}(s)
$$

and called the left (resp. right) stochastic integral of $U_{s}^{*}\left(\right.$ resp. $U_{s}$ ) with respect ot $M_{o}\left(\right.$ resp. $\left.M_{o}^{*}\right)$. While the limits (172) are denoted respectively

$$
\left[\left[M, M^{*}\right]\right](0, t) \quad ; \quad\left[\left[M^{*}, M\right]\right](0, t)
$$

and called the brackets (or the Ito product) of $\mathrm{M}$ with $M^{*}$ (resp. of $M^{*}$ with M) $[6 / d]$.

For example, if $d M=d M^{*}=d W(t)$ with $W(t)$ the Wiener process then we know that

$$
d M^{*} d M=d W^{2} \equiv d t
$$

On the other hand for the Wiener process one has also

$$
E_{t]}\left(d M^{*}(t) d M(t)\right)=E_{t]}\left(d W^{2}(t)\right)=d t
$$

and therefore for the classical Wiener process one has

$$
d M^{*} d M-\sigma(t) d t=d M^{2}-d t \equiv 0
$$


It turns out (cf. [6/d]) that the relation (175) is characteristic of the classical processes with continuous trajectories. For this reason, even if in quantum theory it makes no sense to speak of trajectories of a process, we shall call a continuous trajectory quantum process, any process satisfying condition (175) above. Since the continuity of the trajectories is a basic characteristic of the classical diffusions, we shall say that an equation of the form

$$
d U \equiv(b(t) d t+d M(t)) U_{t}
$$

is of diffusion type if its coefficients satisfy the conditions of Theorem 2 and $d M(t)$ is a continuous trajectory quantum process in the sense just defined. Notice that, if $d M(t)$ has continuous trajectories then (140), (141) become equivalent to:

$$
d M(t)=-d M^{*}(t)
$$

\section{The Forward Langevin equation}

In this Section we study the quantum stochastic analogue of the procedure which leads to the forward Heisenberg equation (35). Let $H,\left(V_{t}^{o}\right)$ and the conditional expectations $\left(E_{t]}\right)$ be given as in Section (2). If $U_{t}$ satisfies the cocycle equation (26) and is adapted to the past filtration then we can define the quantum dynamics

$$
V_{t}=U_{t}^{*} V_{t}^{o}
$$

as in (27) and introduce the forward Heisenberg dynamics as in (31) i.e.

$$
X(t)=V_{t} X V_{t}^{*}=U_{t}^{*} V_{t}^{o} X V_{-t}^{o} U_{t}
$$

(X an observable quantity or, more generally any operator). If $U_{t}$ is differentiable, the equation satisfied by $X(t)$ is the usual Schrödinger equation in Heisenberg form (34). If $V_{t}$ admits only a forward derivative, the equation satisfied by $X(t)$ will be called a (generalized) forward Langevin equation. The term "generalized" refers here to the fact that, up to this moment no markovianity assumption has been made (cf. Definition 4 ). Fixing $t$ and $d t>0$, and starting from the algebraic identity:

$$
d X(t)=d V_{t} X V_{t}^{*}+V_{t} X d V_{t}^{*}+d V_{t} X d V_{t}^{*}
$$


we see using equation (168) for $V_{t}$, that the right hand side of (179) is equivalent to

$$
\begin{gathered}
d X(t) \equiv V_{t} \cdot\left(i\left(H_{o}+H_{I}\right) d t-\frac{1}{2} \sigma(t) d t+d M^{o}(t)\right) \cdot X \cdot V_{t}^{*}+ \\
+V_{t} \cdot X \cdot\left(-i\left(H_{o}+H_{I}\right) d t-\frac{1}{2} \sigma(t) d t+d M^{o *}(t)\right) \cdot V_{t}^{*}+V_{t} \cdot d M^{o}(t) \cdot X \cdot d M^{o *}(t) \cdot V_{t}^{*}
\end{gathered}
$$

Now, in analogy with the deduction of equation (36) in Section (1.), we introduce the quantities:

$$
\begin{gathered}
\tilde{H}_{o}(t)=V_{t} H_{o} V_{t}^{*} \quad ; \quad \tilde{H}_{I}(t)=V_{t} H_{I} V_{t}^{*} \quad ; \quad \tilde{\sigma}(t)=V_{t} \sigma V_{t}^{*} \\
d \tilde{M}^{o}(t)=V_{t} d M^{o}(t) V_{t}^{*}=V_{t} M^{o}(t, t+d t) V_{t}^{*}
\end{gathered}
$$

With these notations, equation (8.4) becomes

$$
\begin{aligned}
& d X(t) \equiv i\left[\tilde{H}_{o}(t)+\tilde{H}_{I}(t), X(t)\right] d t-\frac{1}{2}\{\tilde{\sigma}(t), X(t)\} d t+ \\
& +d \tilde{M}^{o}(t) X(t) d \tilde{M}^{o *}(t)+d \tilde{M}^{o}(t) X(t)+X(t) d \tilde{M}^{*}(t)
\end{aligned}
$$

Using further the decomposition (140), (141) of $d M$, we obtain the equation:

$$
\begin{gathered}
d X(t) \equiv i\left[\tilde{H}_{o}(t)+\tilde{H}_{I}(t), X(t)\right] d t+i\left[d \tilde{N}^{o}(t), X(t)\right]- \\
-1 / 2\{\tilde{\sigma}(t), X(t)\} d t-1 / 2\{d \tilde{P}(t), X(t)\}+d \tilde{M}^{o}(t) X(t) d \tilde{M}^{o *}(t)
\end{gathered}
$$

Where we recognize an Hamiltonian piece with quantum random force $d \tilde{N}(t)$, a damping piece with quantum random force $d \tilde{P}(t)$ and the Ito correction term $d \tilde{M}^{o}(t) X(t) d \tilde{M}^{o} *(t)$. Notice that, if $U_{t}$ satisfies an equation of diffusion type, then $d \tilde{P}(t) \equiv 0$, i.e. the quantum random damping force is present only if the quantum noise of $\mathrm{M}$ has not continuous trajectories in the sense of Section (7.). Equation (184) will be called the (generalized) forward quantum Langevin equation. 


\section{The forward master equation and the quan- tum Feynman-Kac formula}

Let $X$ be an observable and let the quantum dynamics $V_{t}$ be given by (177). In Section (8.) we have introduced the forward quantum Langevin equation as the equation describing the forward Heisenberg evolved of X, i.e.

$$
X(t)=V_{t} X V_{t}^{*}
$$

The equation which describes the conditional expectation (or conditional mean value) of $X(t)$ given the past with respect to a given time origin denoted 0, i.e.

$$
\bar{X}(t)=E_{o]}\left(V_{t} X V_{t}^{*}\right)
$$

is called the forward master (or Fokker-Planck) equation. One would expect that, being $\bar{X}(t)$ the $E_{o]}$ expectation of $X(t)$, its equation could be

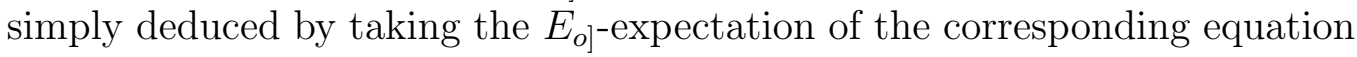
for $X(t)$, i.e. (184). By doing so however, some difficulties arise because it is not clear a priori why by taking the $E_{o}$-expectation of the right hand side of (184), one gets something which is a function of $\bar{X}(t)$. To clarify this point we have to recall briefly the basic idea of the quantum Feynman Kac formula as originally introduced in [1].

A remarkable consequence of the covariance property (??), the Markovian cocycle property and the adaptedness property of Definition 2, is that the 1-parameter family

$$
\bar{X}(t)=P^{t}(X)=E_{o]}(X(t))=E_{o]}\left(V_{t} X V_{t}^{*}\right)
$$

enjoys the semigroup property

$$
P^{s} P^{t}(X)=P^{s+t}(X) \quad ; \quad s, t \geq 0
$$

In fact one has:

$$
P^{s} P^{t}(X)=E_{o]}\left(V_{s} P^{t}(x) V_{s}^{*}\right)=E_{o]}\left(U_{s}^{*} V_{s}^{o} E_{o]}\left(U_{t}^{*} V_{t}^{o} X V_{-t}^{o} U_{t}\right) V_{-s}^{o} U_{s}\right)
$$

in view of the covariance of $E_{t]}$, the right hand side of (189) is equal to

$$
E_{o]}\left(U_{s}^{*} E_{s]}\left[u_{s}^{o}\left(U_{t}^{*}\right) V_{s+t}^{o} X V_{-(s+t)}^{o} u_{s}^{o}\left(U_{t}\right)\right]\right)
$$


by the property of conditional expectations and the adaptedness of $U_{t}$ this is equal to

$$
E_{o]} E_{s]}\left(U_{s}^{*} u_{s}^{o}\left(U_{t}^{*}\right) V_{s+t}^{o} X V_{-(s+t)}^{o} u_{s}^{o}\left(U_{t}\right)\right)
$$

and, by the cocycle property (26) and the projectivity condition (80), this is equal to

$$
E_{o]}\left(U_{s+t}^{*} V_{s+t}^{o} X V_{-(s+t)}^{o} U_{s+t}\right)=P^{s+t}(X)
$$

which proves the semigroup property. The semigroup $P^{t}$ has two important properties:

(i) It is positivity preserving in the sense that, if $\mathrm{X}$ has positive spectrum then, for each $t \geq 0$ also $P^{t}(X)=\bar{X}(t)$ has. (In fact $P^{t}$ has the stronger property of being completely positive (cf. below for a further discussion of this notion).

(ii) It preserves the identity, in the sense that for each $t \geq 0$

$$
P^{t}(1)=1
$$

Any semigroup satisfying conditions (i), (ii) above is called a quantum dynamical semigroup (a quantum Markovian semigroup, in the mathematical literature). Being a semigroup $P^{t}$ has the form

$$
P^{t}=e^{t L}
$$

where $L$ is the infinitesimal generator of $P^{t}$. Therefore the quantity $\bar{X}(t)$, defined by (187) satisfies the linear equation

$$
\frac{d}{d t} \bar{X}(t)=L(\bar{X}(t))
$$

In the classical case the quantity $\bar{X}(t)$ represents the expected value of the observable $X$ at time $t$ given the knowledge of the past of the system (with respect to the time 0 ). In the quantum case this interpretation is possible only in the case in which the range of the conditional expectation $E_{t]}$ is abelian, in fact one cannot obtain a complete information on a non abelian algebra. In the general case $\bar{X}(t)$ represents the partial expectation of $X(t)$ with respect to the noisy degrees of freedom. In many important examples one has to do with a system interacting with a noise in such a way that the corresponding state spaces are kinematically independent, i.e. the state space of the composite system is the tensor product of the state space of the system and the state space of the noise (cf. the example). In these cases, if $X$ is an 
observable of the small system, then $\bar{X}(t)$ represents the reduced evolution of the observable $X$ averaged over the noise space. In any case the equation (192) is called a generalized master equation. The term generalized here is referred to the fact that up to now no Markovianity assumption has been made in the deduction of that equation so that the operator $L$ can in principle depend on the whole past of the system, from $-\infty$ to 0 .

The results of Section (8.) allow to deduce the explicit form of the operator $L$ on the right hand side of the master equation (192). In fact, in order to determine $L$ it will be sufficient, by the semigroup property to evaluate the derivative of $X(t)$ at zero. To this goal consider, for a fixed finite $d t$ the increment

$$
\begin{gathered}
d \bar{X}(t)=\bar{X}(t+d t)-\bar{X}(t)=d E_{o]}(X(t))=E_{o]}(d X(t))= \\
=E_{o]}\left(d V_{t} X V_{t}^{*}\right)+E_{o]}\left(V_{t} X d V_{t}^{*}\right)+E_{o]}\left(d V_{t} X d V_{t}^{*}\right)
\end{gathered}
$$

Using (184) and the martingale property, equation (192) becomes

$$
\begin{gathered}
d \bar{X}(t) \equiv E_{o]}\left(V_{t}\left[i\left(H_{o}+H_{I}\right) d t-\frac{1}{2} \sigma d t+d M^{o}\right] X V_{t}^{*}\right)+ \\
+E_{o]}\left(V_{t} X\left[-\left(i H_{o}+H_{I}\right) d t-\frac{1}{2} \sigma d t+d M^{o *}\right] V_{t}^{*}\right)+E_{o]}\left(V_{t} d M^{o} X d M^{o *} V_{t}^{*}\right)
\end{gathered}
$$

Now we assume that the observable $X$ is localized in $H_{o]}$. On this assumption, equation (194) evaluated at $t=0$, gives, using the fundamental property (97) of $E_{o]}$ and the fact that $X, H_{o}, H_{I}$, are all localized in $H_{o]}$ :

$$
\begin{gathered}
\bar{X}(d t)-\bar{X}(0)=i\left(H_{o}+H_{I}\right) X d t-\frac{1}{2} \sigma X d t+E_{o]}\left(d M^{o}\right) X- \\
-i X\left(H_{o}+H_{I}\right) d t-\frac{1}{2} X \sigma d t+X E_{o]}\left(d M^{o}\right)^{*}+E_{o]}\left(d M^{o} X d M^{o} *\right)
\end{gathered}
$$

Since $E_{o]}\left(d M^{o}\right)=0$ we obtain

$$
\frac{\bar{X}(d t)-\bar{X}(0)}{d t} \equiv i\left[H_{o}+H_{I}, \bar{X}(0)\right]-\frac{1}{2}\{\sigma \bar{X}(0)+\sigma \bar{X}(0) \sigma\}+E_{o]}\left(\frac{d M^{o} X d M^{o *}}{d t}\right)
$$

From (195) we deduce that the limit

$$
\lim _{d t \rightarrow 0^{+}} E_{o]}\left(\frac{d M^{o} X d M^{o *}}{d t}\right)=L_{o}(X)
$$


exists and satisfies:

$$
L_{o}(1)=\sigma
$$

The relation (196) also implies that the map $L_{o}$ is completely positive. This is a strong restriction on $L_{o}$. For example, if $L_{o}: \mathcal{B}(\mathcal{H}) \rightarrow \mathcal{B}(\mathcal{H})$ is a bounded map, then it must have the form

$$
L_{o}(X)=\sum_{j=1}^{\infty} K_{j}^{*} X K_{j}
$$

for some bounded operators $K_{j} \in \mathcal{B}(\mathcal{H})$. From (195) and (196) we conclude that

$$
\begin{gathered}
\left.\quad \frac{d}{d t}\right|_{t=0} \bar{X}(t)=\left.\frac{d}{d t}\right|_{t=0} P^{t}(X)=\left.\frac{d}{d t}\right|_{t=0} e^{t L}(X)= \\
=L(X)=i\left[H_{o}+H_{I}, X\right]-\frac{1}{2}\{\sigma X+X \sigma\}+L_{o}(X)
\end{gathered}
$$

From the general theory of semigroups the relation (199) implies that for each $t$

$$
\frac{d}{d t} \bar{X}(t)=L(\bar{X}(t))=L P^{t}(X)=P^{t}(L(X))
$$

From (199) and (200) we conclude that for each t one has:

$$
\left.\frac{d}{d t} \bar{X}(t)=i\left[H_{o}+H_{I}\right), \bar{X}(t)\right]-\frac{1}{2}\{\sigma \bar{X}(t)+\bar{X}(t) \sigma\}+L_{o}(\bar{X}(t))
$$

or equivalently

$$
\frac{d}{d t} P^{t}(X)=P^{t}\left(i\left[H_{o}+H_{I}, X\right]-\frac{1}{2}\{\sigma, X\}+L_{o}(X)\right)
$$

\section{Remark (9.1).}

The equivalence of (201) and (202) is non trivial because, while (202) is easily deduced from (194), (201) cannot be directly deduced from (194). The situation here is exactly similar to the one met in the elementary deduction of the forward Heisenberg equation in Section (1.) namely: the identity (199) means that the whole operator

$$
L(\cdot)=i\left[H_{o}+H_{I}, \cdot\right]-\frac{1}{2}\{\sigma, \cdot\}+L_{o}(\cdot)
$$

Commutes with $P^{t}$. However this is not true for the individual pieces of the right hand side of (202). We sum up the results of this Section in the following 
Theorem 4 Let $U_{t}$ be a strongly continuous wave operator (cocycle) with respect to the free evolution $u_{t}^{o}(\cdot)=V_{t}^{o}(\cdot) V_{-t}^{o}$ (with $V_{t}^{o}=\exp i t H_{o}$, adapted to the past filtration $\mathcal{H}_{t]}$ and admitting a strongly continuous forward derivative. Then there exist a positive operator $\sigma$, a self-adjoint operator $H_{I}$ and a completely positive map $L_{o}: \mathcal{B}(\mathcal{H}) \rightarrow \mathcal{B}(\mathcal{H})$ satisfying

$$
L_{o}(1)=\sigma
$$

such that, for any observable $X$ localized in $\mathcal{H}_{o]}$, the conditional mean

$$
P^{t}(X)=: \bar{X}(t)=E_{o]}\left(U_{t}^{*} V_{t}^{o} X V_{t}^{o} U_{t}\right)
$$

satisfies the (generalized) forward master equation (201). Moreover the equations (201) and (202) are equivalent.

It is not known if also in the unbounded case the map $L_{o}$ must have the form (198) for some unbounded operators $\left(K_{j}\right)$. However, given a sequence of operators $\left(K_{j}\right)$ such that the expression (198) makes sense on a dense domain, one can define a completely positive map by (198). Such a map will be called in standard form. In the next two sections we show how the Langevin and the master equation look like if the map $L_{o}$ is in standard form. NOTES

If the quantum dynamical semigroup $T_{t}$ is norm continuous, then a theorem of Gorini Kossakowski and Sudarshan [23], and Lindblad [26] shows that its infinitesimal generator $G$ must have the form

$$
G(x)=K^{+} x+x K+\sum_{j=1}^{n} L_{j}^{+} x L_{j} \quad ; \quad x \in \mathcal{A}_{S}
$$

with $L_{j}, K \in \mathcal{A}_{S}$ satisfying

$$
K^{+}+K+\sum_{j=1}^{n} L_{j}^{+} L_{j}=0
$$

Therefore all the (norm continuous) Markovian semigroups can be obtained from the weak or the singular coupling limit by an appropriate choice of the free and interaction Hamiltonians and of the reservoir state. 
There are examples of linear, trace and positivity preserving evolution equations for density operators which are not completely positive.

Complete positivity implies inequalities among the relaxation parameters which are stronger (in general) than the inequalities implied by simple positivity.

The fact that at the moment no experimental violation of the inequalities implied by complete positivity is known and the natural way in which this property arises in the stochastic limit, are good physical reasons to believe that the physical evolutions are completely positive.

Two examples.

1) Two-level systems.

See Section 4 of the paper "Properties of quantum Markovian master equations".

The result is originally due to A. Kossakowski [ref. 51]. Evolutions which are positive but not completely positive might actually arise as semigroup approximations to physical evolutions which are not of semigroup type; see Theorem 7 in the enclosed paper "The averaging method ..."

2) Spin relaxation. See the following papers:

[1] W. Happer: Rev. Mod. Phys. 44 (1972) 169

[2] A. Omont: Progr. Quantum Electronics 5 (1977) 69

[3] M. Verri and V. Gorini: J. Math. Phys. 19 (1978) 1083

[4] V. Gorini, M. Verri and E.C.G. Sudarshan: in Springer Lecture Notes in Physics vol. 135 (1980) 95

The structure of completely positive evolutions for spin relaxation has been studied in [1] and [2] on the basis of models, and in [3] and [4] from a general standpoint. In [3] it was incorrectly concluded that for isotropic spin relaxation there is no distinction between positivity and complete positivity. This has been corrected in [4]. As a simple example, consider isotropic relaxation of a spin $J=1$. Let

$$
T_{K Q}: Q=-K, \ldots K, K=0, \ldots, 2 J(=2)
$$

be the irreducible spherical tensors occurring in the reduction of the repre- 
sentation $D(J) \otimes D(J)$. By rotational invariance,

$$
(d / d t) \operatorname{Tr}\left[\rho_{t} T_{K Q}\right]=-K \operatorname{Tr}\left[\rho_{t} T_{K Q}\right]
$$

In general, $0=0$. The condition for positivity is $0<2<31$, whereas the condition for complete positivity is $(3 / 5) 1<2<31$.

\section{Standard forms of the quantum Langevin equation}

In the previous Section no assumptions on the noise $d M(t)$ were introduced. In this section we study the following problem: Under which conditions on the noise $d M(t)$ will the master equation be in the standard form, i.e. its generator will have the form (198), (199)? The conditions (205), (207) below are sufficient for this to happen. Namely, we require that the noise $d M$ has the form

$$
d M(t)=\sum_{j=1}^{n} u_{t}^{o}\left(F_{j}\right)^{*} d M_{j}(t)
$$

with the $F_{j}$ localized in $\mathcal{H}_{o}$ and the

$$
d M_{j}(t)=M_{j}(t, t+d t)
$$

localized in $\mathcal{H}_{t+d t]}$ and commuting with the past i.e., satisfying

$$
\left[Y(t), M_{j}(t, t+d t)\right]=0
$$

for all $j, t$, all $d t>0$, and any $Y(t)$ localized in $\mathcal{H}_{t]}$. Under these conditions and with the notation

$$
\tilde{F}_{j}(t)=V_{t} F_{j} V_{t}^{*}
$$

we have, using (167), (207), (208) and the unitarity of $U_{t}$ :

$$
d \tilde{M}^{o}(t)=V_{t}\left(u_{-t}^{o}(d M(t))\right) V_{t}^{*}=\tilde{F}_{j}(t)^{*} U_{t}^{*} d M_{j}(t) U_{t}=\tilde{F}_{j}(t)^{*} d M_{j}(t)
$$

Therefore, writing the forward Langevin equation (184) in the form:

$$
d X(t) \equiv i\left[\tilde{H}_{o}(t)+\tilde{H}_{I}(t), X(t)\right] d t+d \tilde{M}^{o} X(t)+X(t) d \tilde{M}^{o *}-
$$




$$
-\frac{1}{2}\left\{d \tilde{M}^{o} d \tilde{M}^{o *}, X(t)\right\}+d \tilde{M}^{o}(t) X(t) d \tilde{M}^{o *}(t)
$$

and using (205), (207), (208), (209) and (210), one easily finds:

$$
\begin{aligned}
d X(t) & \equiv i\left[\tilde{H}_{o}(t)+\tilde{H}_{I}(t), X(t)\right] d t+\sum_{j=1}^{n}\left\{\tilde{F}_{j}(t)^{*} X(t) d M_{j}(t)+X(t) \tilde{F}_{j}(t) d M_{j}^{*}(t)\right\}+ \\
& +\frac{1}{2} \sum_{j, k=1}^{n}\left\{\left[\tilde{F}_{j}^{*}(t), X(t)\right] \tilde{F}_{k}(t)-\tilde{F}_{j}^{*}(t)\left[\tilde{F}_{k}(t), X(t)\right]\right\} d M_{j}(t) d M_{k}^{*}(t)
\end{aligned}
$$

From this equation, taking $E_{o}$-expectations of both sides and using the property $E_{o]} E_{t]}=E_{o}$, the fact that the $F_{j}(t), X(t)$ are localized in $\mathcal{H}_{t]}$, the definition of $\sigma(t)$ i.e.

$$
E_{t]}\left(d M_{j}(t) d M_{k}^{*}(t)\right) \equiv \sigma_{j k}(t) d t
$$

and the fact that $E_{t]}\left(d M_{j}(t)\right)=0$, we finally obtain

$$
d \bar{X}(t) \equiv \begin{gathered}
E_{o]}\left(i\left[\tilde{H}_{o}(t)+\tilde{H}_{I}(t), X(t)\right] d t+\frac{1}{2}\right. \\
E_{o]}\left\{\left[\tilde{F}_{j}^{*}(t), X(t)\right] \tilde{F}_{k}(t)-\tilde{F}_{j}(t)^{*}\left[\tilde{F}_{k}(t), X(t)\right]\right\} \sigma_{j k} d t
\end{gathered}
$$

From the definition (187) of the semigroup $P^{t}$ we see that equation (213) is equivalent to

$$
\frac{d}{d t} P^{t}(X)=P^{t}(L(X))
$$

with $L$ of the form (203), i.e. this is the generator of the forward master equation in standard form obtained in Section (9.). Remark In the diffusion case, characterized by the condition

$$
d M_{j}(t) d M_{k}^{*}(t) \equiv \sigma_{j k}(t) d t
$$

we know from Section (7.) that

$$
d M(t) \equiv-d M(t)^{*}
$$

which, in view of (205), is equivalent to

$$
\sum_{j=1}^{n} u_{t}^{o}\left(F_{j}^{*}\right) d M_{j}(t) \equiv-\sum_{j=1}^{n} u_{t}^{o}\left(F_{j}\right) d M_{j}^{*}(t)
$$


Assuming that the set of $d M_{j}(t)$ is self-adjoint in the sense that for each index $j$ there exists an index, denoted $j^{+}$such that

$$
d M_{j}^{*}(t)=d M_{j^{+}}(t) \quad ; \quad \forall t, d t
$$

then condition (217) is equivalent to

$$
\sum_{j=1}^{n} u_{t}^{o}\left(F_{j}^{*}+F_{j}\right) d M_{j}(t) \equiv 0
$$

which, on the further assumption of independence of the $d M_{j}(t)$ over the past, implies

$$
F_{j}^{*}=-F_{j^{+}} \quad ; \quad j=1, \ldots, n
$$

Therefore in this case the Langevin equation (211) takes the form

$$
\begin{gathered}
d X(t) \equiv i\left[\tilde{H}_{o}(t)+\tilde{H}_{I}(t), X(t)\right] d t+\frac{1}{2}\left\{\left[\tilde{F}_{j}(t)^{*}, X(t)\right] \tilde{F}_{k}(t)+\right. \\
\left.+\tilde{F}_{j}(t)^{*}\left[\tilde{F}_{k}(t), X(t)\right]\right\} \sigma_{j k} d t+\left[\tilde{F}_{j}(t)^{*}, X(t)\right] d M_{j}(t)
\end{gathered}
$$

Remark. Notice that, by separating the self-adjoint and the skew-adjoint part of the $d M_{j}$, i.e. by introducing the new noises

$$
d Q_{j}=\frac{1}{2}\left(d M_{j}+d M_{j}^{*}\right) \quad ; \quad d P_{j}=\frac{1}{2 i}\left(d M_{j}-d M_{j}^{*}\right)
$$

i.e. we can always assume that the noises $d M_{j}$ are self-adjoint

$$
d M_{j}=d M_{j}^{*} \quad ; \quad \forall i=1, \ldots, n
$$

and in this case, on the diffusion assumption (216), the coefficients $F_{j}$ have the form

$$
-i K_{j}=F_{j} \quad ; \quad j=1, \ldots, n
$$

for some self-adjoint operators $K_{j}$. In this case the Langevin equation (183) takes the form

$$
\begin{aligned}
d X(t) & \equiv i\left[\tilde{H}_{o}(t)+\tilde{H}_{I}(t), X(t)\right] d t+\frac{1}{2}\left[\left[\tilde{K}_{j}(t), X(t)\right], \tilde{K}_{k}(t)\right] R e \sigma_{j k} d t+ \\
& +\frac{i}{2}\left\{\left[\tilde{K}_{j}(t), X(t)\right], \tilde{K}_{k}(t)\right\} I m \sigma_{j k} d t+i\left[\tilde{K}_{j}(t), X(t)\right] d M j
\end{aligned}
$$


Or, denoting

$$
L(X)=i\left[H_{o}+H_{I}, X\right]+\frac{1}{2}\left[\left[K_{j}, X\right], K_{k}\right] R e \sigma_{j k}+\frac{i}{2}\left\{\left[K_{j}, X\right], K_{k}\right\} I m \sigma_{j k}
$$

the generator of the Fokker-Plank semigroup one eventually obtains:

$$
d X(t) \equiv V_{t}\left(L(X) V_{t}^{*} d t+i V_{t}\left[K_{j}, X\right] V_{t}^{*} d M_{j}\right.
$$

which, together with (225), makes evident the separation between the linear (double commutator) and the nonlinear (anticommutator) terms. The examples we are going to discuss in the next Sections will take the equations (225), (226) as their starting point.

Two important things have to be remarked concerning the Langevin equation (221): (I.) It is not a single equation, but a system of equations in the unknowns

$$
\tilde{X}(t), \tilde{H}_{o}(t), \tilde{H}_{I}(t), \tilde{F}_{j}(t), \tilde{F}_{j}^{*}(t) \quad ;(j=1, \ldots, n)
$$

with initial conditions

$$
X, H_{o}, H_{I}, F_{j}, F_{j}^{*}
$$

This is clear from the definition (208), (209) of the unknown variables. (II.) It is a nonlinear system, since the products of pairs and even triples of unknowns enter in it. Moreover, given the connections, explained above, between the Langevin equation (221) and the master equation (215), the two remarks above hold also for the associated master equation.

With the change of variables

$$
\begin{gathered}
G_{k}=\sum_{j=1}^{n} u_{k j} F_{j} \\
d N_{j}=\sum_{j=1}^{n} u_{j k} d M_{k}
\end{gathered}
$$

where $U=\left(u_{i j}\right)$ is a unitary matrix diagonalizing $\Sigma=\left(\sigma_{i j}\right)$, equation (211) becomes

$$
\begin{aligned}
& d X(t) \equiv i\left[\tilde{H}_{o}(t)+\tilde{H}_{I}(t), X(t)\right] d t+ \\
& +\frac{1}{2} \gamma_{j}\left\{\left[\quad \tilde{G}_{j}(t)^{*}, X(t)\right] \tilde{G}_{j}(t)-\tilde{G}_{j}(t)^{*}\left[\tilde{G}_{j}(t), X(t)\right]\right\} d t+ \\
& +\quad \tilde{G}_{j}(t)^{*} X(t) d N_{j}(t)+X(t) \tilde{G}_{j}(t) d N_{j}(t)^{*}
\end{aligned}
$$


where the $\gamma_{j}$ are the (positive ) eigenvalues of $\Sigma$. If the $\tilde{G}_{j}=\tilde{G}_{j}(0)=-i K_{j}$ are skew-adjoint then equation (231) becomes

$$
\begin{aligned}
d X(t) \equiv i\left[\tilde{H}_{o}(t)+\tilde{H}_{I}(t), X(t)\right] d t+\frac{1}{2}[ & {\left.\left[\tilde{G}_{j}(t), X(t)\right], \tilde{G}_{j}(t)\right] \gamma_{j} d t+} \\
+ & {\left[X(t), \tilde{G}_{j}(t)\right] d N_{j}(t) }
\end{aligned}
$$

which shows that at least when the $G_{j}$ and $X$ or equivalently, the $F_{j}$ and $X$, are in a finite dimensional Lie algebra, then the nonlinearity depends only on the commutator $\left[\tilde{H}_{o}(t)+\tilde{H}_{I}(t), X(t)\right]$ and therefore, for some special choices of $H_{o}$ and $H_{I}$, might disappear in the new variables $G_{j}$. In Sections (12.) and (13.) we produce two interesting examples where this happens.

\section{Standard forms of the quantum master equation}

In Section (9.) we have deduced the generalized forward master equation (201), i.e.

$$
\frac{d}{d t} \bar{X}(t)=i\left[H_{o}+H_{I}, \bar{X}(t)\right]-\frac{1}{2}\{\sigma, \bar{X}(t)\}+L_{o}(\bar{X}(t))=L(\bar{X}(t))
$$

where $H_{o}, H_{I}$ are self-adjoint operators, $\sigma$ is a positive operator and $L_{o}$ is a completely positive map. In this case the evolution of $\bar{X}(t)$ is described by the quantum dynamical semigroup whose generator is given by (203). The remarkable feature of this equation is that it is independent of the mathematical model of the noise, in the sense that the noise appears in it only through its infinitesimal characteristics $\sigma$ and $L_{o}$. The operators $H_{o}, H_{I}$, act on the past space $H_{o}$ (no Markovianity assumption has been used up to now) and $L_{o}$ is a linear operator mapping the past observables into themselves. If $L_{o}$ has the further property of mapping bounded observables of $H_{o}$ into themselves, then it is known that it must have the form

$$
L_{o}(Y)=\sum_{j=1}^{n} G_{j}^{*} Y G_{j}
$$

where $\mathrm{n}$ is a natural integer or $+\infty$ and the $G_{j}$ are bounded operators acting on $H_{o}$. This result shows that the form (2) of $L_{o}$ is a generic one and in this 
section we shall study how the forward master equation (234) looks like if the map $L_{o}(Y)$ has the form (235). In fact we shall assume that $L_{o}$ has the slightly more general form.

$$
L_{o}(Y)=\sum_{j, k=1}^{n} F_{j}^{*} Y F_{k} \sigma_{j k}
$$

where the $F_{j}$ are operators acting on $H_{o]}$ and $\left(\sigma_{j k}\right)=\Sigma$ is a complex matrix of positive type. If $n<+\infty$, then the two forms (235) and (236) are equivalent because in this case $\Sigma$ has the form

$$
\Sigma=U^{*} \Lambda U \Leftrightarrow \sigma_{i j}=\sum_{k=1}^{n} \bar{u}_{k i} \gamma_{k} u_{k j}
$$

for some unitary matrix $U=\left(u_{i j}\right)$ on some diagonal matrix $\Lambda=\left(\gamma_{k}\right)$ with positive elements. Therefore with the change of variables

$$
G_{k}=\sum_{j=1}^{n} \sqrt{\lambda}_{k} u_{k j} F_{j} \quad ; \quad k=1, \ldots, n
$$

(236) becomes (235). However, even if mathematically equivalent, the two forms (235) and (236) are not physically equivalent because in several examples the $F_{j}$ might have a simple direct physical interpretation but not the $G_{k}$. Assuming that $L_{o}$ has the form (236), the generator $L$ of the quantum dynamical semigroup, describing the evolution of $X(t)$, is given by

$$
L(X)=i\left[H_{o}+H_{I}, X\right]-\frac{1}{2} \sum_{j, k=1}^{n}\left\{F_{j}^{*} F_{k}, X\right\} \sigma_{j k}+\sum_{j, k=1}^{n} F_{j}^{*} X F_{k} \sigma_{j k}
$$

where, as usual, $[\cdot, \cdot]$ denotes the commutator and $\{\cdot, \cdot\}$ the anticommutator. Using the identity

$$
-\frac{1}{2}\left\{F_{j}^{*} F_{k}, X\right\}+F_{j}^{*} X F_{k}=\frac{1}{2}\left[F_{j}^{*}, X\right] F_{k}-\frac{1}{2} F_{j}^{*}\left[F_{k}, X\right]
$$

and separating the real and the imaginary part of $\sigma_{j k}$, one finds (since $\bar{\sigma}_{j k}=$ $\left.\sigma_{k j}\right)$ :

$$
L(X)=i\left[H_{o}+H_{I}, X\right]+\frac{1}{2} \sum_{j, k}\left\{\left[F_{j}^{*}, X\right] F_{k}-F_{k}^{*}\left[F_{j}, X\right]\right\} \sigma_{j k}=
$$




$$
\begin{aligned}
=i\left[H_{o}+H_{I}, X\right]+\frac{1}{2} \quad & \sum_{j \leq k}\left\{\left[F_{j}^{*}, X\right] F_{k}-F_{k}^{*}\left[F_{j}, X\right]\right\} R e \sigma_{j k}+ \\
& +\frac{i}{2} \sum_{j \leq k}\left\{\left[F_{j}^{*}, X\right] F_{k}+F_{k}^{*}\left[F_{j}, X\right]\right\} I m \sigma_{j k}
\end{aligned}
$$

A better insight into the meaning of the generator is given by considering some particular cases: if all the $F_{j}$ are self-adjoint, then

$$
F_{j}^{*}=F_{j} \quad ; \quad j=1, \ldots, n
$$

then $L$ takes the form

$$
L(X)=i\left[H_{o}+H_{I}, X\right]+\frac{1}{2}\left[\left[F_{j}, X\right], F_{k}\right] R e \sigma_{j k}+\frac{i}{2}\left\{\left[F_{j}, X\right], F_{k}\right\} I m \sigma_{j k}
$$

The same result, i.e. (11.10), is obtained if all the $F_{j}$ are skew-adjoint

$$
F_{j}^{*}=-F_{j}
$$

If moreover the covariance $\Sigma$ is diagonal, i.e.

$$
\sigma_{i j}=\gamma_{j} \delta_{i j}
$$

then the generator takes the form

$$
L(X)=i\left[H_{o}+H_{I}, X\right]+\frac{1}{2} \sum_{j} \gamma_{j}\left[\left[F_{j}, X\right], F_{j}\right]
$$

and, when $\mathrm{X}$ is taken to be one of the $F_{j}$ and the $F_{j}$ are the generators of a Lie algebra, we obtain the standard form of a quantum diffusion which corresponds to a linear Langevin equation. It is not true however that, with the change of variables (229), (230) any master equation can be brought to the form (246). In fact such a change of variables will bring to an equation of the form

$$
L(X)=i\left[H_{o}+H_{I}, X\right]+\frac{1}{2} \sum_{j} \gamma_{j}\left(\left[G_{j}^{*}, X\right] \cdot G_{j}-G_{j}^{*} \cdot\left[G_{j}, X\right]\right)
$$

which reduces to (246) only if the $G_{j}$ are self-adjoint. 


\section{The damped harmonic oscillator}

Consider the Langevin equation (??) in the case of two independent selfadjoint noises $M_{1}, M_{2}$ with coefficients

$$
K_{1}=a q \quad ; \quad K_{2}=b p
$$

where $q, p$ are the position and momentum operators of a representation of the Heisenberg commutation relation

$$
[q, p]=i
$$

and $a, b$ are real constants. Suppose moreover that

$$
\left[H_{o}, p\right]=\left[H_{o}, q\right]=0
$$

i.e. the free evolution acts trivially on $p$ and $q$, and choose $H_{I}$ to be the usual harmonic oscillator hamiltonian

$$
H_{I}=\frac{1}{2}\left(p^{2}+q^{2}\right)
$$

Assuming that the noises $M_{1}, M_{2}$ have a conditional covariance of the form

$$
E_{o]}\left(\frac{d M_{i}(t) d M_{j}(t)}{d t}\right)=\sigma_{i j}
$$

where the $\sigma_{i j}$ are complex numbers, one easily derives the action of the generator of the master equation on $p, q$ :

$$
L(p)=-q-p a b \operatorname{Im} \sigma_{12} \quad ; \quad L(q)=p-q a b \operatorname{Im} \sigma_{12}
$$

with $L$ given by (239). Denoting

$$
\gamma=a b \operatorname{Im} \sigma_{12} \in \mathbf{R}
$$

the forward master equation for $\bar{p}(t), \bar{q}(t)$ (defined by (187)) becomes

$$
\begin{gathered}
\frac{d}{d t} \bar{q}(t)=\bar{p}(t)-\gamma \bar{q}(t) \\
\frac{d}{d t} \bar{p}(t)=-\bar{q}(t)-\gamma \bar{p}(t)
\end{gathered}
$$


Notice that the present theory predicts that, even introducing an asymmetry in the interaction of $p$ and $q$ with the noise (cf. (248) above) the damping constant for $\bar{p}(t)$ and $\bar{q}(t)$ will be the same.

The forward Langevin equation (??) becomes in our case

$$
\begin{gathered}
d q(t) \equiv(p(t)-\gamma q(t)) d t+b d M_{2} \\
d p(t) \equiv(-q(t)-\gamma p(t)) d t-a d M_{1}
\end{gathered}
$$

And one recognizes the Senitzky-Lax [33], [22] linear quantum Langevin equations for the position and momentum observables of a damped harmonic oscillator except for the fact that we have not yet specified the form of the noises $d M_{1}, d M_{2}$. However, the unitarity of the evolution imposes that, for all times $t$ one has

$$
0=d[q(t), p(t)]=[d q(t), p(t)]+[q(t), d p(t)]+[d q(t), d p(t)]
$$

which, in view of $(12.9),(12.10)$ (and with $a=b=1$ ) is equivalent to

$0 \equiv\left[(p(t)-\gamma q(t)) d t+d M_{2}, p(t)\right]+\left[q(t),\left(-q(t)-\gamma p(t) d t-d M_{1}\right]+\left[d M_{2}, d M_{1}\right]\right.$

and, since according to (207) the increments $d M_{j}$ commute with the past i.e.

$$
\left[d M_{2}, p(t)\right]=\left[q(t), d M_{1}\right]=0
$$

this is equivalent to

$$
\left[d M_{1}, d M_{2}\right] \equiv 2 i \gamma d t
$$

Because of the commutativity with the past, one has $\left[d M_{1}, d M_{2}\right]=d\left[M_{1}, M_{2}\right]$. Therefore the identity (258) can be integrated yielding

$$
\left[M_{1}(t), M_{2}(t)\right] \equiv 2 i \gamma t
$$

Using again the commutativity with the past one finds that (259) is equivalent to

$$
\left[M_{1}(s), M_{2}(t)\right] \equiv 2 i \gamma s \wedge t
$$

This shows that, on the assumption that the increments of the noise commute with the past, the commutation relations, postulated by Lax [22] for the quantum Brownian motion, are not only a sufficient, but also a necessary condition for the preservation of the Heisenberg commutation relations. 


\section{The angular momentum Lie algebra}

We now consider the Langevin equation (221) in the case of three independent noises $M_{1}, M_{2}, M_{3}$ whose coefficients satisfy the angular momentum commutation relations

$$
\left[J_{\alpha}, J_{\beta}\right]=i \epsilon_{\alpha \beta \gamma} J_{\gamma} \quad ; \quad \alpha, \beta, \gamma=1,2,3
$$

where $\epsilon_{\alpha \beta \gamma}(\alpha, \beta, \gamma=1,2,3)$ denotes the completely antisymmetric symbol with $\epsilon_{123}=1$. In order to write down the quantum Langevin system for the observables $J_{1}(t), J_{2}(t), J_{3}(t)$, we have to substitute these observables for the unknown $X(t)$ in the general Langevin equation (221). Evaluating, on these assumptions, the commutators and the anticommutators and again in the assumption that the free Hamiltonian $H_{o}$ commutes with the coefficients of the noises, i.e.

$$
\left[H_{o}, J_{k}\right]=0 \quad ; \quad k=1,2,3
$$

we obtain

$$
\begin{aligned}
d J_{1} & =i\left[J_{1}, H\right] d t+i(J \wedge d M)_{1}+\frac{1}{2}\left(-J_{1}\left(\sigma_{22}+\sigma_{33}\right)+J_{2} R e \sigma_{12}+J_{3} R e \sigma_{13}\right) d t+ \\
+ & \left(\frac{1}{2}\left(I m \sigma_{13}\right)\left\{J_{1}, J_{2}\right\}-\frac{1}{2}\left(I_{1 m} \sigma_{12}\right)\left\{J_{1}, J_{3}\right\}+\left(I m \sigma_{23}\right)\left(J_{2}^{2}+J_{3}^{2}\right)\right) d t \\
d J_{2} & =i\left[J_{2}, H\right] d t+i(J \wedge d M)_{2}+\frac{1}{2}\left(-J_{2}\left(\sigma_{11}+\sigma_{33}\right)+J_{1} R e \sigma_{12}+J_{3} R e \sigma_{23}\right) d t+ \\
+ & \left(-\frac{1}{2}\left(I m \sigma_{12}\right)\left\{J_{2}, J_{3}\right\}-\frac{1}{2}\left(I m \sigma_{23}\right)\left\{J_{2}, J_{1}\right\}-\left(I m \sigma_{13}\right)\left(J_{1}^{2}+J_{3}^{2}\right)\right) d t \\
d J_{3} & =i\left[J_{3}, H\right] d t+i(J \wedge d M)_{3}+\frac{1}{2}\left(-J_{3}\left(\sigma_{11}+\sigma_{22}\right)+J_{2} R e \sigma_{23}+J_{1} R e \sigma_{13}\right) d t+ \\
& +\left(\frac{1}{2}\left(I m \sigma_{13}\right)\left\{J_{3}, J_{2}\right\}-\frac{1}{2}\left(I m \sigma_{23}\right)\left\{J_{3}, J_{1}\right\}-\left(I m \sigma_{12}\right)\left(J_{1}^{2}+J_{2}^{2}\right)\right) d t
\end{aligned}
$$

where $J=\left(J_{1}, J_{2}, J_{3}\right), d M=\left(d M_{1}, d M_{2}, d M_{3}\right.$ and $(J \wedge d M)_{1}=\left(J_{2} d M_{3}-\right.$ $\left.J_{3} d M_{2}\right)$ and similarly for the ther consequents. Thus, if we choose for $H_{1}$ the Hamiltonian of the free rotator:

$$
H_{I}=J_{1}^{2}+J_{2}^{2}+J_{3}^{2}
$$

the Hamiltonian terms in the above equations are reduced to the only contribution of the random force. 
This Langevin system is in general nonlinear in the unknowns $J_{1}(t), J_{2}(t), J_{3}(t)$ . Only in the case in which the imaginary part of the covariance of the noise vanishes we obtain some linear equations. The meaning of these equations is best understood in terms of the (conditionally ) averaged observables

$$
\bar{J}_{k}(t)=E_{0]}\left(J_{k}(t)\right)
$$

In fact, by taking $E_{0]}$-expectations of both sides of the equations (262), (263), (264), one obtains:

$$
\begin{aligned}
\frac{d}{d t} \bar{J}_{1} & =\frac{1}{2}\left(-\bar{J}_{1}\left(\sigma_{22}+\sigma_{33}\right)+\bar{J}_{2} R e \sigma_{12}+\bar{J}_{3} R e \sigma_{13}\right) \\
\frac{d}{d t} \bar{J}_{2} & =\frac{1}{2}\left(-\bar{J}_{2}\left(\sigma_{11}+\sigma_{33}\right)+\bar{J}_{1} \operatorname{Re} \sigma_{12}+\bar{J}_{3} R e \sigma_{23}\right) \\
\frac{d}{d t} \bar{J}_{3} & =\frac{1}{2}\left(-\bar{J}_{3}\left(\sigma_{11}+\sigma_{22}\right)+\bar{J}_{2} \operatorname{Re} \sigma_{23}+\bar{J}_{1} \operatorname{Re} \sigma_{13}\right)
\end{aligned}
$$

Which are precisely the Bloch equation in standard form [13]. Notice that our deduction provides a microscopic interpretation of the constants in the Bloch equations in terms of the covariance of the noises.

Recalling the definition 224 of the $\sigma_{j k}$, we see that the fact that the imaginary part of the $\sigma_{j k}$ are nonzero is due to the fact that terms of the form $d M_{j}^{*} d M_{k}$ are of order $d t$. If we have a single noise $d M$ (and its adjoint ), this reduces to the nonvanishing (up to $o(d t)$ ) of the terms of the form $d M d M$. If, as in most known examples, $M$ is an annihilation process, then this event corresponds to a nonzero probability of creating (or annihilating ) two quanta of the noise field in an infinitesimal time interval $d t$. This phenomenon is due to the non gauge invariance of the noise $M$ hence it is justified to call it a squeezing effect (for the experimental differences, induced on the solutions of the master equation by these squeezing effects, cf. [6/a]). 


\section{References}

[1] Accardi L. On the quantum Feynman-Kac formula. Rend. Sem. Mat. Fis. Univ. Polit. Milano, 48 (1978) 135-179

[2] Accardi L., A.Frigerio , J.T.Lewis Quantum stochastic processes. Publications RIMS 18 (1982) 97-133

[3] Accardi L., Quantum Stochastic Calculus. in: Proceedings 4-th Vilnius Conference on Probability and Mathematical Statistics. VNU Press 1986

[4] Accardi L. A mathematical theory of quantum noise in: Proc. Ist World Congress of the Bernoulli Society eds.Yu. Prohorov, V.V. Sazonov, vol. 1, pg.427-444, VNU Science Press

[5] Accardi, L. A.Frigerio and Lu Yun-Gang (1988): On the weak coupling limit. To appear in : Quantum Probability and Applications IV, Springer LNM

[6] Accardi, L., Frigerio, A., Lu Y.G.: The weak coupling limit in the finite temperature case. to appear

[7] Accardi L. , Abundo M. Squeezing corrections to the Bloch equations. Preprint 1988

[8] Accardi L., Bach A. Central limits of squeezing operators. to appear in : Quantum Probability and Applications IV Springer LNM

[9] Accardi L. , Fagnola F., Quaegebeur J. Quantum Stochastic Calculus preprint 1989

[10] Accardi L., Quaegebeur J. The Fermion Levy martingale representation theorem. submitted to: J.Funct Anal.

[11] Accardi L., R.Hudson The non abelian cohomology of stochastic derivations to appear in : Quantum Probability and Applications V Springer LNM

[12] Applebaum D. Stochastic dilations of the Bloch equations in boson and fermion noise. J.Phys. A: Math. Gen. 19 (1986) 937-959

[13] Bloch F. Phys.Rev. 70 (1946) 460 
[14] Bloch F., Wangness R.K. Phys.Rev. 89 (1953) 728

[15] Arecchi F.T. Instabilities and chaos in single mode homogeneous line lasers. in: "Instabilities and chaos in quantum optics" F.T.Arecchi and R.G. Harrison eds., Springer 1984

[16] Dellacherie M., Meyer P.A. Probabilites et potentiel. Hermann (1975)

[17] Doob J.L. Stochastic Processes. John Wiley 1967 7.)Emch G.G. Positivity of the K-entropy of non-abelian K-flows. Z.Wahrsch. Verw. Geb. 29 (1974) 241-252

[18] Emch G.C , Varilly J.C. On the standard form of the Bloch equation. Comm. Math.Phys. 3 (1979) 113-116

[19] Fagnola F. Quantum stochastic calculus and a Boson Levy theorem To appear in : Quantum Probability and Applications IV, Springer LNM

[20] Gorini, V., Kossakowski, A., and Sudarshan, E.C.G. (1976): Completely positive dynamical semigroups on N-level systems. J. Math. Phys. 17, $821-825$.

[21] Ford W., Kac M., Mazur J. J.Math. Phys. 6 (1965) 504

[22] Haken H. Laser theory. Springer 1984

[23] Hasegawa H.,Klauder J.R., Lakshman M. Towards a satisfactory formulation of the quantum Langevin equation. J.Phys.A (Math. Gen.) 18 (1985) L123-L128

[24] Hudson R.L., Streater R.F. Ito's formula is the chain rule with Wick ordering. Phys. Letters 86A (1981) 277-279

[25] Hudson R.L., Parthasarathy K.R. Quantum Ito's formula and stochastic evolutions. Comm. Math. Phys. 93 (1984) 301-323

[26] Kerner E.H. Can. Journ. Phys. 36 (1958) 371

[27] Kubo R. J.Phys.Soc. Japan 12 (1957) 571 J.Phys.Soc. Japan 26 (1969) Suppl1-5

[28] Lax M. Quantum noise theory of noise sources. Phys. Rev. 145 (1966) $110-129$ 
[29] Lewis J.T., Thomas L.C. On the existence of a class of stationary quantum stochastic Processes. Ann. Ist. Henri Poincare' 22 (1975) 241-248

[30] Lewis J.T., H.Maassen Hamiltonian models of classical and quantum stochastic processes. in: Quantum Probability and applications to the quantum theory of irreversible processes. eds. Accardi L., Frigerio A., Gorini V. Spri nger LNM 1055

[31] Lindblad, G. (1976): On the generators of quantum dynamical semigroups. Commu. Math. Phys. 48, 119-130.

[32] Masani P. Orthogonally scattered measures. Advances in Math. 2 (1968) $61-117$

[33] Nakazawa H. Quantization of Brownian motion process in potential field. in: Quantum probability and applications II. Springer LNM 1136

[34] Nelson E. Dynamical Theories of Brownian motion. Princeton University Press 1972

[35] Palmer, P.F. (1977): J. Math. Phys. 18, 527-529.

[36] Pule J.V. The Bloch equations. Comm. Math. phys. 38 (1974) 241-256

[37] Schwinger J. Quantum kinematics and dynamics. Academic Press 1970

[38] Schröder W. $W^{*}$-K-systems. Dissertation Tubingen 1983

[39] Senitzky I.R. Dissipation in Quantum Mechanics. The harmonic oscillator. Phys. Rev. 119 (1960) 670

[40] Stevens W.K.H. Proc. Phys. Soc.(London) 72 (1958) 1027

[41] Streater R.F. Damped oscillator with quantum noise. J.Phys. A (Math. Gen.) 15 (1982) 1477-1485

[42] von Waldenfels Wilhelm Ito solution of the linear quantum stochastic differential equation describing light emission and absorption. in: Quantum probability and applications to the quantum theory of irreversible processes. Springer LNM 1055

[43] Wolf D. (Ed): Noise in Physical Systems. Springer-Verlag, 1978. 
[44] Kon M.A.: Probability Distributions in Quantum Statistical Mechanics. Springer-Verlag, 1985.

[45] Malchow H., Schimansky-Geier L.: Noise and Diffusion in Bistable Nonequilibrium Systems. Teubner-Texte zur Physik.

[46] Steeb W.-H., Louw J.A.: Chaos and quantum chaos. World Scientific, 1986.

[47] Surjan P.S.: Second quantization approach to Quantum Chemistry. Springer, 1989.

[48] Scott S. Chemical chaos. Oxford, 1991. 